\title{
Clinical Ultrafast Laser Surgery: Recent Advances and Future Directions
}

\author{
Christopher L. Hoy, Onur Ferhanoğlu, Murat Yildirim, Ki Hyun Kim, Sandeep S. Karajanagi, \\ Ka Man Carmen Chan, James B. Kobler, Steven M. Zeitels, and Adela Ben-Yakar
}

(Invited Paper)

\begin{abstract}
Ultrafast pulsed lasers can be used to achieve remarkable precision during surgical ablation. Through nonlinear interactions with tissue, ultrafast lasers can provide a largely non-thermal mechanism of ablation and a unique ability to create targeted damage within bulk tissue. These advantages have made ultrafast lasers the ideal surgical tool for various novel applications in ophthalmology. Clinical adoption of ultrafast lasers in other surgical applications remains limited in part due to the lack of a means for fiber delivery of ultrafast laser pulses as a flexible, hand-held surgical endoscope. This review provides an overview of the recent advances in bringing this unique surgical tool into the clinic. We discuss fundamental mechanisms and limitations of ultrafast laser ablation, novel techniques for overcoming these limitations, the current state of clinical applications, and conclude with our recent efforts in developing fiber-coupled probes for flexible ultrafast laser surgery and imaging.
\end{abstract}

Index Terms-laser ablation, surgery, nonlinear optics, biomedical optical imaging.

\section{INTRODUCTION}

A LMOST since their inception, lasers have been put to use as versatile biological cutting tools. The invention of the laser in 1960 intrigued biologists and clinicians alike with the prospect of a surgical tool capable of creating targeted damage with diffraction-limited precision [1], [2]. Quickly thereafter, a variety of laser surgery applications were developed, ranging in scale from sub-cellular dissection of organelles [3]-[5] and chromosomes [6] to bulk tissue ablation of eyes [7], skin [8],

Manuscript received July 16, 2013; revised August 29, 2013; accepted August 30, 2013. Date of current version December 12, 2013. This work was supported by the National Science Foundation under Grants BES-0548673, CBET-1014953, and Career Award CBET-0846868, as well as a grant from the Texas Ignition Fund by the University of Texas Board of Regents.

C. L. Hoy is with the Department of Mechanical Engineering, The University of Texas at Austin, Austin, TX 78712 USA and also with the Center for Optical Diagnostics and Therapy, Department of Radiation Oncology, Erasmus Medical Center, Rotterdam, The Netherlands (e-mail: c.1hoy@erasmusmc.nl).

O. Ferhanoğlu, M. Yildirim, K. H. Kim, and A. Ben-Yakar are with the Department of Mechanical Engineering, The University of Texas at Austin, Austin, TX 78712 USA (e-mail: oferhanoglu@utexas.edu; yildirim@utexas.edu; kihyunkim@utexas.edu; ben-yakar@mail.utexas.edu).

S. S. Karajanagi, K. M. C. Chan, J. B. Kobler, and S. M. Zeitels are with the Center for Laryngeal Surgery and Voice Rehabilitation, Massachusetts General Hospital, Boston, MA 02114 USA (e-mail: skarajanagi@ partners.org; kaman@ mit.edu; James.Kobler@mgh.harvard.edu; zeitels.steven@mgh.harvard.edu).

Color versions of one or more of the figures in this paper are available online at http://ieeexplore.ieee.org.

Digital Object Identifier 10.1109/JSTQE.2013.2287098 and teeth [9]. Over the next 40 years, the surgical applications of lasers grew and matured, leading to an abundance of discoveries in cell biology [10] and clinical applications throughout medicine, where acceptance has been particularly strong in ophthalmology [11], dermatology [12], and otolaryngology [13].

The development of clinical laser techniques has centered on continuous wave (cw) and nanosecond or longer pulsed lasers. These conventional laser surgery techniques rely predominantly on linear absorption of laser light because the laser intensities are generally too low to induce appreciable nonlinear interaction at practical average powers. Owing to the linear absorption mechanism, photodamage from these lasers is highly wavelength-dependent and thermal in nature [14]. This wavelength dependence can be exploited to create a tissue-selective effect. However it can also result in non-deterministic cutting effects when cutting heterogeneous tissue and can limit efficacy in transparent or low-absorbing samples. Similarly, though laser heating has been used to great effect clinically for both cauterization of laser incisions and tissue welding [15], the diffusion of heat away from the laser focal volume can lead to collateral damage outside the focal volume and may lead to scarring in biological tissues. While both wavelength-dependence and heating can be mitigated or exploited, the linear absorption of laser light throughout the laser-tissue interaction volume leads to increased damage outside the focal volume along the laser path. This lack of axial confinement ultimately limits their precision inside thicker specimens. With the development of utlrafastpulsed laser sources in the early 1980s, which deliver pulse durations in the range of $100 \mathrm{fs}$ to $10 \mathrm{ps}$, biologists and clinicians were given access to new predominantly non-thermal regimes of photodamage, which have increased surgical precision to the diffraction limit and beyond.

Thus far, the clinical potential of ultrafast laser microsurgery has barely begun to be realized, with clinical adoption limited to ophthalmic applications. One of the technological barriers to the adoption of this technology is the lack of a means to flexibly deliver the laser light to clinical sites in or on the patient. The goal of this review is to provide the reader with a perspective on both the current state of clinical ultrafast laser surgery development as well as insights into future directions. To that end, the review begins with a brief introduction to the fundamental mechanisms and limitations of femtosecond laser ablation, which provide the constraints that dictate potential areas of application. Here we discuss the fundamental boundaries to the maximum ablation depth and novel strategies to mitigate these limitations. 
TABLE I

Review of Ultrafast Laser Microsurgery Mechanisms. AdAPTed From Ben-YaKar and Bourgeois [24]

\begin{tabular}{clcc}
\hline $\begin{array}{c}\text { Damage } \\
\text { mechanism }\end{array}$ & Photochemical Damage & $\begin{array}{c}\text { Thermoelastic Stress } \\
\text { Confinement }\end{array}$ & Optical Breakdown \\
\hline $\begin{array}{c}\text { Intensity } \\
\text { threshold }\end{array}$ & $0.26 \times 10^{12} \mathrm{~W} / \mathrm{cm}^{2}$ & $5.1 \times 10^{12} \mathrm{~W} / \mathrm{cm}^{2}$ & $6.54 \times 10^{12} \mathrm{~W} / \mathrm{cm}^{2}$ \\
\hline $\begin{array}{c}\text { Electron } \\
\text { density at } \\
\text { threshold }\end{array}$ & $\begin{array}{l}2.1 \times 10^{13} \mathrm{~cm}^{-3} \\
\text { One free electron in the } \\
\text { focal volume }\end{array}$ & $\begin{array}{l}0.24 \times 10^{21} \mathrm{~cm}^{-3} \\
\text { Induced thermal stress } \\
\text { overcomes the tensile } \\
\text { strength of water }\end{array}$ & $\begin{array}{l}1.0 \times 10^{21} \mathrm{~cm}^{-3} \\
\text { Critical electron } \\
\text { density for optical } \\
\text { breakdown }\end{array}$ \\
\hline Description & $\begin{array}{l}\text { Free electrons } \\
\text { participate in chemical } \\
\text { reactions to form } \\
\text { destructive reactive } \\
\text { oxygen species and lead } \\
\text { to breaking of chemical } \\
\text { bonds. }\end{array}$ & $\begin{array}{l}\text { Thermalization of the } \\
\text { plasma occurs faster than } \\
\text { the acoustic relaxation time. } \\
\text { Confinement of thermal } \\
\text { stresses leads to formation } \\
\text { of nano-scale transient } \\
\text { bubbles. }\end{array}$ & $\begin{array}{l}\text { Damage is preated by } \\
\text { temperature plasma and } \\
\text { by the accompanying } \\
\text { shock wave and } \\
\text { cavitation bubble. }\end{array}$ \\
\hline $\begin{array}{c}\text { Pulse } \\
\text { repetition rate }\end{array}$ & $\begin{array}{l}\text { A large number of } \\
\text { pulses are required. For } \\
\text { practical reasons high } \\
\text { repetition rate lasers are } \\
\text { preferable. }\end{array}$ & $\begin{array}{l}\text { Bubble lifetime is 100-500ns. At higher repetition } \\
\text { rates and higher irradiances, heat accumulation and } \\
\text { long lasting bubble formation can become significant. }\end{array}$ \\
\hline
\end{tabular}

We continue with a summary of the current state of clinical ultrafast laser surgery development in both soft and hard tissues. Lastly, we conclude with details of our recent efforts toward developing a new clinical application, the treatment of scarred vocal folds, and the creation of a fiber-coupled clinical tool capable of delivering femtosecond laser pulses to medical regions of interest for both microsurgery and microscopic visualization of the microsurgery site.

\section{MECHANISMS AND TECHNICAL CONSIDERATIONS IN ULTRAFAST LASER SURGERY}

Surgery with focused ultrafast lasers is triggered by the generation of an initial population of free electrons through a combination of multiphoton ionization and band-gap (Zener) tunneling [16], [17]. These ionization pathways are nonlinearly dependent on the local light intensity and thus the region of free electron generation is highly-localized in three dimensions within the focal volume. The free electrons may then act as seed electrons for avalanche ionization, wherein an initial free electron is accelerated by the incident laser field and collides with a bound valence electron, thus ionizing the bound electron through impact ionization. This doubling process can then repeat, causing a cascade, until termination of the laser pulse or total ionization of the valence electron population. The final density of this exponentially growing number of free electrons determines the precise mechanism of damage, which can be dominated by photochemical effects, thermoelastic bubble nucleation, or optical breakdown. While the relative contributions of these ionization pathways can vary with pulse duration, the fundamental characteristics of ultrafast laser ablation have been shown to be fairly consistent for pulse durations up to 5-10 ps [16], [18]. Each of the three mechanisms are summarized in Table I and will be discussed briefly below, while the interested reader can find further details in the work of Vogel et al. [17] and Joglekar et al. [16].

Photochemical damage can occur at relatively low irradiances. Vogel et al. theoretically modeled that photochemical effects may begin at peak laser irradiances as low as $0.26 \mathrm{TW} / \mathrm{cm}^{2}$ [17], which corresponds to one free electron generated in the focal volume of a $1.3 \mathrm{NA}$ objective lens per pulse ${ }^{1}$. In the photochemical damage pathway, energetic free electrons may either 1) cause damage through disassociation of water molecules, leading to the creation of reactive oxygen species (ROS) that can destroy cellular components [19], or 2) participate directly in the bond breaking of other cellular components [20]. It is also possible that non-ionizing multiphoton absorption may aid photochemical damage in structures such as DNA, where the simultaneous absorption of three near-infrared (NIR) photons may be sufficient to cause direct bond breaking due to the widely observed single-photon absorption peak for $260 \mathrm{~nm}$ light [21]. Because creation of appreciable photochemical damage requires a large number of relatively low energy pulses, high-repetition rate $(\sim 100 \mathrm{MHz})$ ultrafast oscillators are required to create useful damage in a reasonable amount of time.

Thermoelastic damage can occur at peak laser irradiances exceeding approximately $5.1 \mathrm{TW} / \mathrm{cm}^{2}$. In this regime, thermalization of the free electrons leads to rapid, confined heating inside the focal volume. The subsequent thermoelastic stresses generated therein can lead to the formation of transient nanoscale bubbles [17]. Bubble lifetimes near the nucleation threshold are on the order of tens to hundreds of nanoseconds. Because the thermalization of the electrons occurs over four orders of

\footnotetext{
${ }^{1}$ Unless otherwise specified, the threshold irradiances provided here assume $100 \mathrm{fs}$ pulses with $800 \mathrm{~nm}$ center wavelength focused into water by a $1.3 \mathrm{NA}$ lens, in accordance with the model used in Vogel et al [17]. Peak irradiance damage thresholds in the femtosecond regime have been shown to be only weakly dependent on these parameters, however.
} 
magnitude faster than the characteristic thermal diffusion time, temperature effects are well confined and it is believed to be the nucleation and expansion of the nanoscale bubble that is responsible for damage in this regime. For ultrafast laser surgery in this regime, amplified laser systems at lower repetition rates are used, both due to the higher pulse energies required and the need to prevent accumulative pulse effects from increasing the extent of damage.

Lastly, damage due to optical breakdown can occur when laser irradiances above approximately $6.5 \mathrm{TW} / \mathrm{cm}^{2}$ are delivered and the critical free electron density of $\sim 10^{21} \mathrm{~cm}^{-3}$ can be met and exceeded. At this point, the plasma at the focal volume becomes highly absorbing and the remaining pulse energy acts primarily to increase the plasma energy density. In this ablation regime, the rapid ionization of the focal volume is accompanied by formation of a cavitation bubble and emission of a shock wave, which may induce mechanical damage to the target that extends beyond the focal volume. While optical breakdown can also be induced using up to nanosecond pulse durations, seed electrons for avalanche ionization generally must be provided by linear heating and thermionic emission. Because ultrafast laser pulses can produce their own seed electrons through multiphoton ionization and tunneling, ablation with ultrafast laser pulses can be conducted in the bulk of transparent and minimally-absorbing materials, such as the transparent tissues of the eye.

For peak irradiances above the photochemical damage threshold, multiple pulses in a pulse train can be used to lower the damage threshold through a repetition-rate dependent incubation effect [22], [23], which can be useful for creating optical breakdown without unwanted nonlinear effects, such as selffocusing effects described below, that can effect pulse propagation. Each of the aforementioned damage mechanisms relies on the same rapid generation of free electrons via the nonlinear optical interaction with matter described earlier. As a result of the nonlinear mechanism, ultrafast laser damage in dielectrics has been generally shown to exhibit only a weak dependence on wavelength in the approximate wavelength range of 500 $1500 \mathrm{~nm}$ [17], [25].

\section{Fundamental Limitations of ABLATION DEPTH INSIDE TISSUE AND METHODS FOR OVERCOMING THESE LIMITATIONS}

The high peak intensities of ultrafast laser pulses enable the nonlinear interaction with tissue that permits confined ablation inside bulk tissue. However, these high peak intensities also introduce unwanted nonlinear effects that can limit the maximum penetration depth and precision of ablation.

When using ultrafast laser pulses for microsurgery within bulk tissue, the primary limitation to the maximum ablation depth and precision is ultimately the phenomenon of selffocusing. Self-focusing with ultrafast laser pulses occurs when the intense laser pulses create a spatial variation in the refractive index profile via the nonlinear index of refraction, $n_{2}$, and the Kerr effect [26], [27]. The Kerr effect arises from the third order nonlinear susceptibility and is thus present to some degree in all materials. As a result, the index of refraction in a Kerr medium is given by

$$
n=n_{0}+n_{2} I(r, t)
$$

where $n$ is the total index of refraction, $n_{0}$ is the linear index of refraction, and $I(r, t)$ is the irradiance as a function of beam radius, $r$, and time, $t$. Representative values for $n_{2}$ are approximately $5.4 \times 10^{-16} \mathrm{~cm}^{2} / \mathrm{W}$ for water at $804 \mathrm{~nm} \mathrm{[28]} \mathrm{and}$ $2.0 \times 10^{-15} \mathrm{~cm}^{2} / \mathrm{W}$ for porcine cornea at $1030 \mathrm{~nm}$ [29]. Thus for irradiances delivered during ultrafast laser ablation, the nonlinear component of the refractive index becomes significant and spatially varying, creating the effect of a positive lens. For a collimated laser beam propagating in a dielectric Kerr medium, the self-focusing effect competes with diffraction, with the two phenomena balancing each other when the peak power of the pulse is approximately [30]

$$
P_{c r}=\frac{0.471 \lambda^{2}}{\pi n_{0} n_{2}}
$$

where $P_{c r}$ is the critical power for self-focusing and $\lambda$ is wavelength. Beyond this critical power, self-focusing dominates and the pulse begins to collapse and focus before the geometric focus.

For focused pulses the critical power is approximately the same, where the distance to beam collapse is simply shifted by the lens transformation. Due to the temporal evolution of the pulse, self-focusing laser pulses exhibit a "moving focus" [30], wherein the low intensity front and tail of the pulse will focus toward the geometric focus whilst the high-intensity peak of the pulse will focus earlier. In practical cases, such catastrophic self-focusing is often arrested by a combination of factors, such as plasma defocusing from generated free electrons, self-phase modulation and subsequent dispersion reducing pulse intensity, and reduction of pulse energy due to scattering and absorption in turbid media [31]. Nevertheless, self-focusing effects can lead to material modification both leading up to and behind the geometric focus when intense pulses are focused inside bulk material [32]-[35]. Though generally detrimental, this beam filamentation phenomenon can be exploited in some cases, for example in delivering targeted ionizing radiation in tumor therapy [36].

When focusing inside scattering tissue, increasing laser energy is required to reach the damage threshold at the focus as the focus is moved deeper into the tissue due to attenuation following the Beer-Lambert law. Thus as depth is increased, the peak power of the incoming laser pulses are exponentially increased until $P \gg P_{c r}$ and catastrophic self-focusing shifts the focus significantly shallower than the targeted depth.

Fortunately, several approaches can be taken to mitigate the effects of self-focusing in surgical applications. First, because self-focusing depends only on the peak power of the pulse, focusing with a higher numerical aperture (NA) objective can help avoid self-focusing while still achieving optical breakdown. For Corning 0211 glass, which has a critical power at $800 \mathrm{~nm}$ of approximately 1.5 MW, Schaffer et al. found self-focusing effects to be negligible when focusing with NAs above 0.9 [37]. The critical power in this study was very similar to the critical power of 1.2 MW found by Miclea et al. for porcine cornea at 
$1030 \mathrm{~nm}$ [29]. It should be noted, however, that while higher NA can help to avoid the onset of self-focusing, it can also lead to increased spherical aberrations when focusing deep into tissue without use of immersion fluid to match the index of refraction of the tissue [38]. Spherical aberration enlarges the focal volume, particularly in the axial direction, degrading the confinement of ablation.

Second, for pulse durations below approximately 10 ps, optical breakdown occurs without significant free electron recombination during the pulse and the threshold fluence becomes only weakly dependent on pulse duration [17], [18]. In practice, this weak dependence implies that increasingly shorter pulse durations will serve to increase self-focusing, without a commensurate decrease in ablation threshold. For example, a $100 \mathrm{fs}$ pulse $^{2}$ with a sech ${ }^{2}$ temporal profile, a critical power of $1.2 \mathrm{MW}$ is achieved with only $140 \mathrm{~nJ}$ pulse energy, while a 3 ps pulse can contain $4.2 \mu \mathrm{J}$ of energy before reaching the same peak power. Thus pulse durations on the order of hundreds of femtoseconds to few picoseconds may be useful to avoid self-focusing effects while preserving damage confinement.

Third, the pulse-to-pulse incubation effects previously mentioned can provide a reduced damage threshold when multiple pulses are delivered during ablation at repetition rates in the $\mathrm{kHz}$ regime and above. Subsequently, a series of pulses can be used to create optical breakdown in bulk tissue while keeping the individual pulse peak power well below the critical power for self-focusing.

In summary, to maximize the practical ablation depth with ultrashort pulsed lasers, it is beneficial to 1) maximize the focusing NA while 2) utilizing pulse durations in the hundreds of femtoseconds or low single picoseconds, and 3) using repetition rates in the hundreds of kilohertz where pulse-to-pulse accumulation effects can be utilized without introducing heating or significantly increasing procedure time.

Recently, two novel methods have been proposed that can potentially increase the photodisruption depths inside turbid tissue. The first method, developed by Ben-Yakar and colleagues, proposes to use the near-field enhancement of plasmonic nanoparticles to reduce thresholds for photodisruption [39]-[41]. In this technique, the laser field is strongly enhanced in the nearfield of the nanoparticles when the incident laser frequency coincides with the plasmon frequency of the metal nanoparticles, causing the particles to act as "nanolenses." We have demonstrated a near-field enhancement of 23- and 35-fold during ablation of a silicon surface functionalized with nanoparticles [40] and photodisruption of cells labelled with nanoparticles, respectively [41]. Due to the near-field enhancement of the metal nanoparticles, tissue ablation can be performed with much lower peak power levels which in return helps avoiding the onset of self-focusing. For successful application of this technique, the metal-nanoparticles need to be delivered in vivo to the location of ablation, either topically or systemically, and careful attention must be paid to the targeting specificity and clearance of the nanoparticles.

\footnotetext{
${ }^{2}$ Note that all pulse durations in this document are measured at the full width at half maximum (FWHM).
}

The second method involves temporal focusing techniques, which have been used to decrease the peak powers of weakly focused laser pulses as they propagate through tissue, thus avoiding self-focusing effects. In simultaneous spatial and temporal focusing (SSTF), a dispersive element such as a grating is used to spatially separate the spectral components of the ultrafast laser pulse. The resulting pulse is temporally as well as spatially dispersed, resulting in a dramatically reduced peak intensity. Focusing by the objective lens serves to spatially confine the spectrally dispersed beam back to a diffraction-limited spot, thus recombining the spectral pulse components and restoring the ultrafast pulse duration at the focus. The reduced peak power of the pulse during propagation reduces undesirable non-linear effects such as self-focusing and filamentation, thereby improving the maximum ablation depth and axial confinement. Using the SSTF technique, Durst et al. have experimentally demonstrated temporal focusing of a 1.64 ps pulse at $275 \mu \mathrm{m}$ from the focal plane down to $84 \mathrm{fs}$ at the focal plane [42]. The use of SSTF to avoid self-focusing has been experimentally demonstrated in ultrafast laser optical breakdown in water [43] and in micromachining [44], [45]. The clinical relevance of SSTF has only recently begun to be explored, with Block et al. demonstrating SSTF for ablation in porcine lenses [46]. In Block et al., the authors observed that use of SSTF eliminated nonlinear effects such as focal shift, self-focusing, and filamentation, and exhibited enhanced ablation confinement.

\section{ApPliCATiOnS OF Ultrafast LASER SURGERY}

In comparison with nanosecond-pulsed and cw laser ablation of biological tissues, numerous early studies of ultrafast laser ablation have found improvements in the ablated surface quality and decreased regions of collateral damage [47]-[50]. These qualities, coupled with the ability to create confined damage within bulk tissue, have spurred interest in the use of ultrafast lasers as clinical tools for surgery in a variety of applications and tissue types.

\section{A. Ophthalmology}

By far, the most significant clinical development and acceptance of ultrafast laser surgery has occurred in the field of ophthalmology, specifically in the application of "blade-free" femtosecond laser-assisted in situ keratomileusis (fs-LASIK). In conventional LASIK surgery, a microkeratome is used to cut a thin flap of a thickness of several hundred microns or less on the cornea. This flap is then pulled back to expose the underlying stroma, at which point an excimer laser is used to ablate the corneal stroma, reshaping it to correct aberrations and improve visual acuity. In fs-LASIK, the femtosecond laser is used to create the flap instead of a microkeratome, thereby improving uniformity and predictability of flap thickness and reducing the incidence of complications [51], [52]. A comparison of a fsLASIK flap and a traditional LASIK flap is provided in Fig. 1(a) and (b). First clinically demonstrated in 2003 [51] and marketed by IntraLase Corp. (now owned by Abbott Medical Optics), the fs-LASIK procedure has gained wide-spread acceptance with femtosecond lasers being used in $30 \%$ of all LASIK procedures 

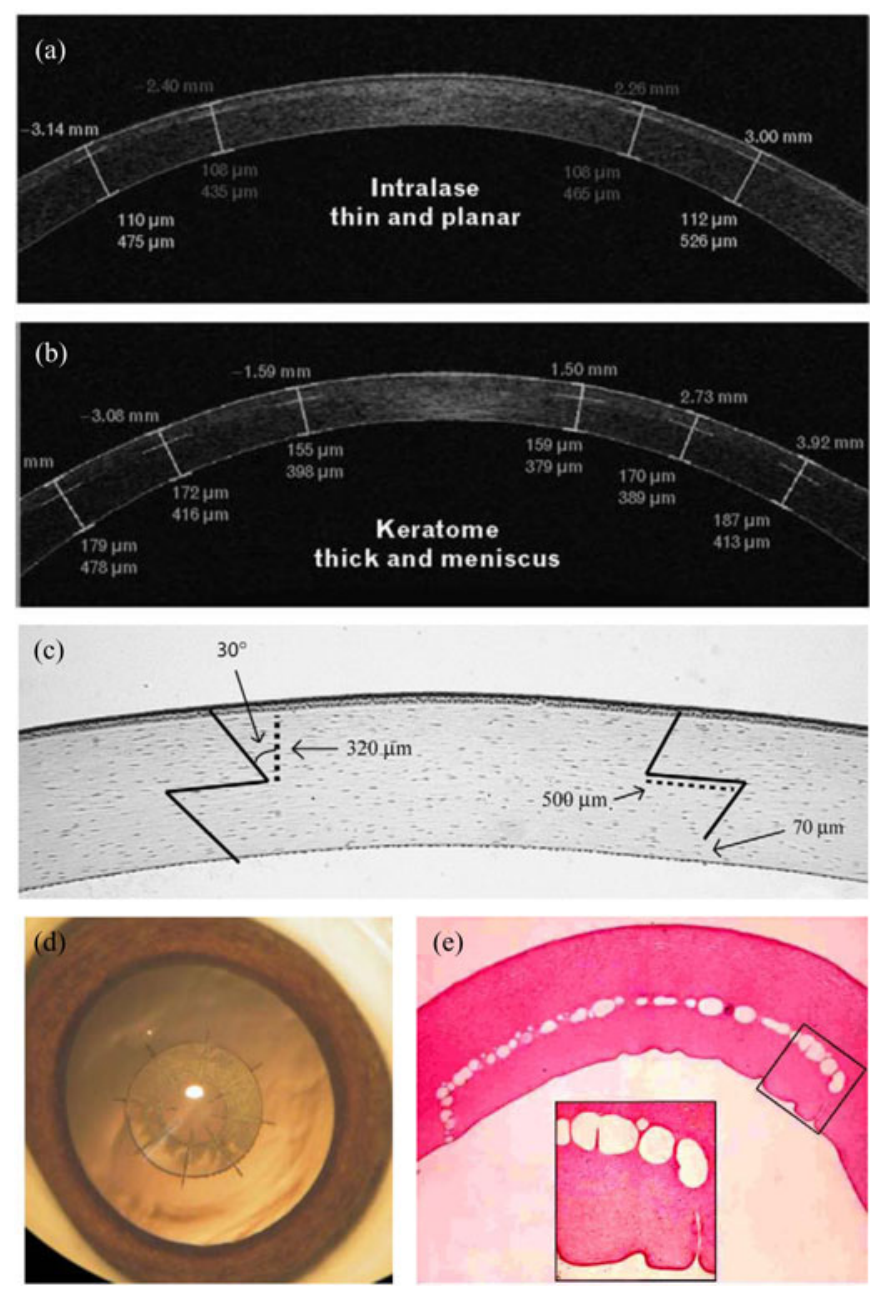

Fig. 1. Representative images of ophthalmic ultrafast laser microsurgery applications. Optical coherence tomography images of LASIK flaps created with, (a) the IntraLase femtosecond laser and, (b) a conventional keratome [52]. The flap is the superficial layer on the cross-section shown. Note that the IntraLase flap is thinner with better uniformity of thickness. (c) Optical image of a donor cornea seated in the eye with a proposed "zig-zag" interface that can be created by ultrafast laser pulses [60]. (d) Light microscopy image of the spoked-wheel pattern cut in the bulk of the lens to produce "gliding planes" in the proposed presbyopia treatment [75]. (e) Histological section of a posterior lamellar graft cut in a porcine cornea [56]. Note that in the applications shown in (c)-(e), the ultrafast laser has enabled complex and/or sub-surface ablations not practical with conventional methods.

by 2006 [53]. Recently, several other commercial ultrafast laser systems have been developed for ophthalmology [54] and fsLASIK remains the most widely-accepted clinical adaptation of ultrafast laser surgery. This successful translation may be because fs-LASIK 1) targets a region of interest that is easily accessible without the need for fiber optic delivery, 2) capitalizes on the ultrafast laser's strength of making precise cuts within transparent bulk tissue, which uniquely enables the ultrafast laser to replace the microkeratome, 3) provides sufficient improvement in outcome to offset the additional cost, and 4) integrates into a pre-existing laser surgery procedure which avoids major changes in the way the surgical tool interfaces with the patient or with the physician, speeding development and acceptance.
In addition to the success of fs-LASIK, and in large part because of this success, a number of other ophthalmological applications for ultrafast lasers are currently being pursued. Among these, the use of ultrafast lasers for keratoplasty (corneal transplantation) has undergone rapid development and entered clinical practice [55]. In keratoplasty, either a partial thickness (anterior or posterior lamellar keratoplasty) or the entire thickness (penetrating keratoplasty) is removed from the patient's cornea and replaced by tissue from a donor eye. For this procedure, ultrafast lasers have been demonstrated to cut the tissue in both donor and recipient eyes in ex vivo animal and human studies since 2003 [56], in in vivo animal studies since 2006 [57], and in vivo in human patients since 2007 [58]. Compared to a conventional blade, ultrafast lasers offer the advantages of high-reproducibility of cut dimensions and the ability to more easily cut increasingly complex shapes [59], [60] for increased donor-to-recipient tissue surface interaction [Fig. 1(d) and (e).] Recent studies indicate that ultrafast laser-assisted keratoplasty can result in improved wound healing time [61] and reduced astigmatism [58]. Interested readers may find more details about the use of ultrafast lasers in specific types of keratoplasty in the review by Soong and Malta [62] and the references therein.

A number of other ophthalmological applications of ultrafast laser surgery are either being developed or are in the early stages of clinical acceptance. These applications include the use of ultrafast lasers to:

1) replace continuous curvilinear capsulorhexis during cataract surgery [63]-[66],

2) create intracorneal tunnels for the implantation of PMMA ring segments [51], [67]-[70],

3 ) excise segments of corneal tissue for diagnostic corneal biopsy [71], [72],

4) produce arcuate cuts inside the cornea to correct astigmatism [73],

5) cut planes inside the eye to increase deformability for treatment of presbyopia [Fig. 1(c)] [74]-[77], and

6) create pockets for intracorneal implantation of keratoprosthesis [78].

In these applications, with the exceptions of the proposed presbyopia treatment, the ultrafast laser is used to replace mechanical cutting tools, thereby increasing precision and repeatability while reducing the frequency of complications.

\section{B. Soft Tissue Ablation Outside of Ophthalmology}

In addition to ophthalmological applications, ultrafast laser microsurgery techniques have been investigated in other soft tissues. In skin, early bench-top studies have indicated that ultrafast lasers can ablate dermal tissue without any observable collateral damage to surrounding tissue [79], [80]. Frederickson et al. investigated surface ablation of excised rat dermis and found that thermal damage effects were avoided when using fewer pulses delivered to the focal spot and energies close to the ablation threshold [79]. Conversely, delivery of 35 pulses at $10 \mathrm{~Hz}$ repetition rate and pulse energy over ten times the threshold energy led to thermal damage extending $30 \mu \mathrm{m}$ in this case [79]. The authors propose that this small amount of thermal 
damage could be intentionally induced to provide hemostasis for in vivo ablation of the dermis, though superficial ablation with thermal collateral damage of this scale is also achievable with less expensive conventional laser sources.

Though dermal tissues are highly scattering compared to the tissues of the eye, several studies have also demonstrated confined sub-surface ultrafast laser ablation in the epithelium and dermis without causing damage to the superficial cellular structure [35], [80], [81]. Specifically, Tse et al. succeeding in ablating porcine skin up to $1 \mathrm{~mm}$ below the tissue surface as verified by acoustic wavefield measurements [35]. The conditions used in this study resulted in significant self-focusing effects, however, degrading the axial confinement of the laser damage as evidenced by bubble formation detected above and below the focal plane. Further study is warranted to explore the optimum conditions for maximum ablation depth in the absence of selffocusing.

Aside from these initial bench-top investigations, there has been limited development of dermatological applications for ultrafast laser ablation, despite the potential for improved wound healing, reduced scar formation, and improved cosmetic results. Though intended for use with excised tissue, recent studies by Huang and Guo demonstrated the successful use of ablation with femtosecond laser pulses to separate layers of excised dermis for use in skin grafts [82], [83]. This laser surgery method demonstrated improved precision over manual layer separation which reduced tissue waste and allowed many layers to be separated from a single strip of donor dermal tissue.

In addition to dermal tissue, several studies investigated clinical application of ultrafast neurosurgery in the late 1990s [49], [84], culminating in the design of a hollow stereotactic probe for delivery of ultrafast laser pulses into the human brain [85], [86]. The probe utilizes concentric hollow tubes to deliver the laser light and steer the focused beam through a cylindrical focal plane around the probe. Several applications within neurosurgery have been suggested, such as ablation in the third ventricle to relieve hydrocephalus, partial ablation of the thalamus to relieve tremors associated with Parkinson's disease, or the resection of small tumors [49]. More recently, the Schaffer group at Cornell University has demonstrated the use of ultrafast laser ablation to delay and attenuate seizure propagation in mouse models of focal epilepsy [87]. Despite the early interest and development, ultrafast laser ablation of neural tissues is still in infancy. With increasing availability of ultrafast laser systems and further development of ultrafast laser microsurgical tools, new applications may develop as neurosurgeons gain wider access to this technology.

\section{Hard Tissue Ablation}

In addition to ablation of soft tissues, ultrafast lasers have found numerous applications in ablation of bone and teeth as well. Dental applications of laser ablation have been widely pursued and a number of techniques have achieved a limited degree of clinical acceptance [88]. The driving force behind the development of non-contact laser dental tools has been the desire to avoid the pain and patient discomfort associated with conventional dentistry tools. Laser dental tools can also provide the potential for material-selective ablation by exploiting differences in absorption spectra, thereby limiting the removal of healthy tissue [89], [90]. Initially, development of laser dentistry tools was hampered by the heating of dental pulp leading to tooth necrosis [91]. Even with the use of Er:YAG lasers, which typically emit at $2.94 \mu \mathrm{m}$, which have greatly reduced heating for ablation of dentin and enamel [92], the increased procedure time required for laser dentistry has limited acceptance [93]. Conventional dental laser systems are also unable to provide the quality of surface finish required for some procedures, such as the preparation of crown stumps [88]. Lastly, many lasers and conventional diamond burr rotary instruments have been shown to produce micro cracks during material removal, which can weaken the tooth and increase susceptibility to future dental caries [94]-[96].

Encouragingly, early studies of ultrafast laser ablation of both dentine and enamel have found a greatly reduced thermal load while eliminating the formation of micro cracks [50], [97]-[99]. Notably, Rode et al. measured the intrapupal temperature of a tooth during $200 \mathrm{~s}$ of surface ablation by $\sim 100 \mathrm{fs}$ NIR pulses at $1 \mathrm{kHz}$ repetition rate [98]. The authors found that, while the increase in pupal temperatures could exceed the damage threshold of $5.5^{\circ} \mathrm{C}$ [91], the use of air cooling at $5 \mathrm{l} / \mathrm{min}$ or higher kept pupal heating at safe levels. By comparison, Neev et al. found a negligible temperature increase below $2.5^{\circ} \mathrm{C}$ at the back of a 1-mm thick dentin slab during ablation with similar pulses at $10 \mathrm{~Hz}$ [50]. These results suggest that in the kilohertz repetition rate regime or above, care must still be taken to ensure safe internal temperature levels are not exceeded. When paired with a cooling, repetition rates in the tens to hundreds of kilohertz are still desirable to achieve satisfactory procedure times. Using a $45 \mathrm{kHz}$ repetition rate, Niemz et al. demonstrated ablation rates comparable to Er:YAG lasers and slow-speed mechanical drills [100]. Use of newer fiber laser systems operating at hundreds of kilohertz repetition rate should decrease procedures times even further.

While the use of ultrafast pulsed lasers for dental ablation can reduce the cracking and thermal loading associated with conventional laser systems, the weak wavelength dependence of ultrafast laser ablation threshold means that much of the inherent selectivity between caries (cavities) and healthy dentin is lost. To regain this selectivity, Serbin et al. have proposed a means of monitoring the spectra from the plasma created during optical breakdown to identify the material being ablated, which could be tied into a feedback mechanism to control the laser exposure [102].

In addition to teeth, ultrafast lasers have been applied to hard tissue ablation of bone. The use of lasers in lieu of mechanical tools for bone surgery is attractive because of the potential for increased precision and the potential to access surgical sites with less invasiveness. Compared with conventional lasers, the lack of hemostasis that accompanies ultrafast laser ablation may provide fewer complications in bone surgery compared to procedures in more vascularized tissues.

Using conventional lasers, laser ablation of bone is predominantly accompanied by thermal damage, often evidenced by 

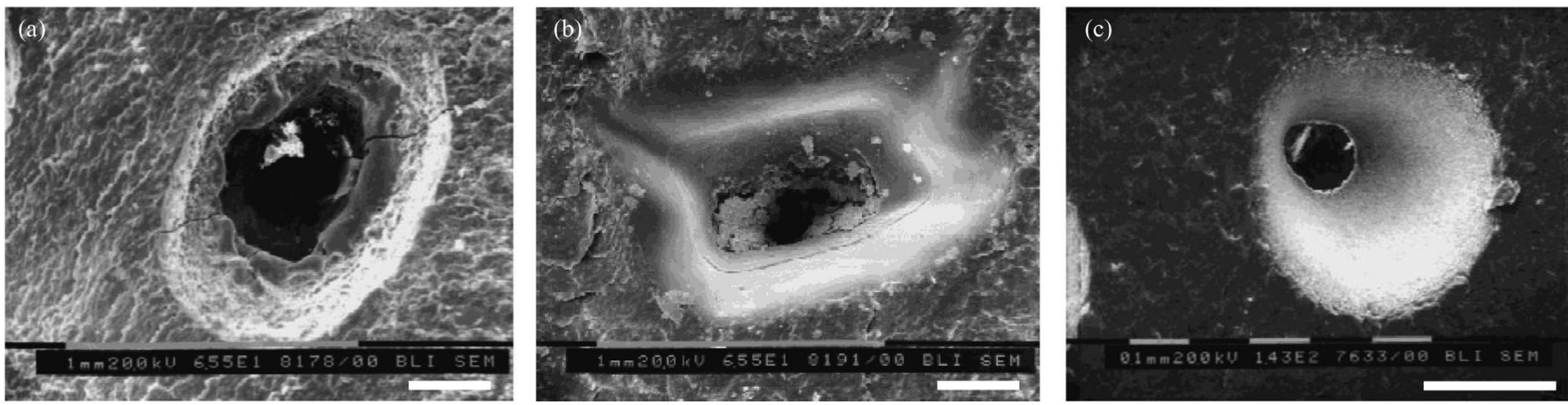

Fig. 2. Scanning electron micrographs of laser ablation in human nail bed. Craters ablated with 100 pulses using (a) Ho:YSGG $(\lambda=2080 \mathrm{~nm}, \tau=250 \mu \mathrm{s})$ and (b) $\mathrm{XeCl}(\lambda=308 \mathrm{~nm}, \tau=15 \mathrm{~ns})$ using $8-24 \mathrm{~J} / \mathrm{cm}^{2}$ with a $1 \mathrm{~mm}^{2}$ focal spot (exact fluence per pulse is not reported) (c) Crater ablated with 100 Ti:sapphire $\left(\lambda=1053 \mathrm{~nm}, \tau=350 \mathrm{fs}\right.$ ) laser pulses at $2 \mathrm{~J} / \mathrm{cm}^{2}$ with a $0.2 \mathrm{~mm}^{2}$ spot size. Note that the thermally affected areas and cracking noticeable in (a) are not found in (b) or (c). Adapted from [101]. Scale bars are $250 \mu \mathrm{m}$

carbonization, and, in the case of pulsed lasers, the generation of significant mechanical stress waves [14]. A delay in the onset of wound healing has been noted in conventional laser ablation of bone and attributed to the thermal damage zone [103]. In addition, acoustic stresses can lead to localized cracking and potentially cause hearing damage in laser surgeries of the middle ear bones [104], an area in which laser ablation has great potential due to the high precision and non-contact nature of laser tools.

Early studies of femtosecond laser ablation in bone and nail tissue showed no observable thermal damage or cracking, as shown in Fig. 2 [50], [101]. Furthermore, in a detailed examination of extracellular and intracellular enzymatic activity on cultured bone tissue after ablation with femtosecond and nanosecond laser pulses, Girard et al. observed a dramatic decrease in enzyme denaturation using femtosecond laser pulses [105]. Spectroscopic analysis of the surface of pure hydroxyapatite (a major bone constituent) also showed no change in chemical composition after near-threshold ablation with femtosecond laser pulses [106]. Despite the observed reduction in collateral damage effects, however, a slight delay in wound healing has still been observed in mouse skulls ablated with femtosecond lasers when compared to mechanical tools [107]. In the middle ear, bench-top studies have found femtosecond lasers to improve ablation surface quality for stapedotomy while reducing thermal and mechanical collateral damage compared to conventional lasers [108]-[110].

For practical clinical use, ultrafast lasers must be able to provide material removal at speeds comparable to conventional techniques. Several studies have found the rate of ablation per pulse achieved by femtosecond lasers in hard tissue to be approximately $1 \mu \mathrm{m} /$ pulse when using near $2 \mathrm{~J} / \mathrm{cm}^{2}$ peak fluence at a $10 \mathrm{~Hz}$ repetition rate [50], [101], [108], compared to rates of approximately 2,7 , and $30 \mu \mathrm{m} /$ pulse found in human nail for $\mathrm{Ho}: Y S G G, \mathrm{XeCl}$, and Er:YAG lasers, at $4 \mathrm{~Hz}$ repetition rate and peak fluences of approximately 24,12 , and $16 \mathrm{~J} / \mathrm{cm}^{2}$, respectively [101].

From this study, the Er:YAG laser (2940 nm wavelength, $250 \mu$ s pulse) appears the best choice among the lasers investigated for applications which require high ablation speeds but do not have stringent requirements on cracking and morphol- ogy. The femtosecond laser pulses from the Ti:sapphire system (1053 nm wavelength, 350 fs pulse) achieved the highest ablation efficiency, implying that very little energy was wasted as heat or mechanical shock wave. However, in Fig. 2(b), the craters created by the $\mathrm{XeCl}$ laser ( $308 \mathrm{~nm}$ wavelength, $15 \mathrm{~ns}$ pulse) appeared to match the quality of the femtosecond laser-created craters while providing a seven-fold increase in ablation rate for the parameters investigated. The comparable ablation quality indicates that nanosecond laser pulses in the near-ultraviolet (UV) can suffice for precise superficial ablation of hard tissue. Indeed, the excellent surface quality during superficial ablation is why excimer lasers similar to the $\mathrm{XeCl}$ laser remain the tool of choice for reshaping the corneal stroma during LASIK, despite the use of femtosecond lasers to create the Bowman's flap to expose the corneal stroma. However, the ablation efficiency of the nanosecond $\mathrm{XeCl}$ pulses was found to be an order of magnitude lower than the femtosecond Ti:sapphire pulses. The excess energy in the nanosecond $\mathrm{XeCl}$ laser ablation is converted into shock waves [111], which can be damaging to hearing in otological applications and may make ultrafast lasers a more appropriate tool for ablation in the middle ear.

While the ultrafast laser proved to provide the slowest per pulse ablation rate in the study discussed above, the slightly lower per pulse ablation rate of ultrafast lasers can be overcome with higher repetition rates and correspondingly higher average powers. Liu and Niemz investigated using femtosecond lasers to cut through femoral bone using a pulse repetition rate of $40 \mathrm{kHz}$ [112]. Based on these experiments, the authors estimate that a cut through the femur for knee replacement surgery would take approximately $20 \mathrm{~min}$. While the authors cite this speed as meeting clinical expectations, further decreases in procedure time can be gained through further increasing the pulse repetition rate.

Recently, femtosecond lasers have been investigated as a means to treat kidney stones via laser lithotripsy of urinary calculi [113]. In this study, Qiu et al. found that very high energy $(640 \mu \mathrm{J})$ femtosecond pulses focused with 0.40 NA could ablate urinary calculi in a bench-top microscope. Compared to nanosecond laser lithotripsy with a Ho:YAG laser, the ultrafast laser produced debris that was one to two orders of magnitude smaller and greatly reduced the mechanical shock waves 
generated in the calculi. Recently the authors have presented ablation of calculi using a hollow core multimode fiber [114]. The fiber has an inner core on the order of $1 \mathrm{~mm}$ in diameter and can deliver $\sim 200$ fs pulses with pulse energies of at least $700 \mu \mathrm{J}$ [115]. While the delivery of such high energy pulses can be beneficial for developing flexible endoscopic microsurgery applications, the modal quality, dispersion, and attenuation of this fiber are all highly sensitive to bending, which may prove difficult to manage in clinical practice.

The clinical applications discussed in this section, both proposed and realized, take advantage of an increased precision and/or the ability to create confined effects inside bulk tissue. To capitalize on the benefits of increased precision or to target structures in bulk tissue, the microsurgery must have a means of guidance and frequently require a flexible means of laser delivery.

\section{APPLICATION OF ULTRAFAST LASER MiCROSURGERY FOR SCARRED VOCAL FOLDS}

As previously mentioned, the barriers to clinical acceptance of ultrafast laser surgery are lower in applications that leverage the unique ability of ultrafast lasers to create precise cuts inside bulk tissue due to the lack of competing technologies. Similarly, the barriers to clinical acceptance are also lower in applications for which there is no accepted treatment with conventional methods. For these reasons, we have been developing a technique for treating scarred vocal folds wherein focused ultrafast laser pulses create a sub-epithelial space in scarred tissue, which enables localization of injected biomaterials to restore the desired mechanical properties [116].

Vocal fold scarring is a common side effect of surgical treatment of laryngeal cancer and can also result from disease or prolonged mechanical stress. The presence of scar tissue in the vocal folds increases their stiffness, thus degrading or even eliminating voice function (phonation). Current treatment options are very limited. A variety of injected biomaterials have been suggested for restoring the viscoelasticity of the vocal folds [117]. However optimal localization of the material within the scarred tissue is likely to be extremely difficult and unpredictable with injection alone because the injected material tends to follow the path of least resistance, ending up where it is least needed. To address this challenge, we have proposed a treatment in which an injection space is created through sub-epithelial ablation of a planar region in the vocal fold. In a bench-top study on ex vivo porcine vocal folds, we have succeeded in creating precisely targeted sub-epithelial voids using 750 fs pulses with 500-nJ pulse energy from a $500 \mathrm{kHz}$ fiber laser, shown in Fig. 3. The use of a high repetition rate amplified fiber laser also enabled surgical guidance via second harmonic generation (SHG) from collagen using the same laser source, and represents a significant decrease in cost, size, and complexity over the laser oscillator and chirped pulse amplifier combination frequently used for nonlinear imaging and surgery.

More recently, we have successfully demonstrated localization of a polyethylene glycol- (PEG) based biomaterial stained with Rhodamine dye into the ablated sub-epithelial void of

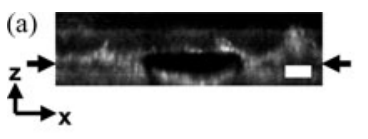

(b)

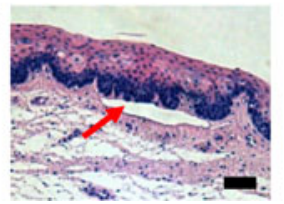

(c)

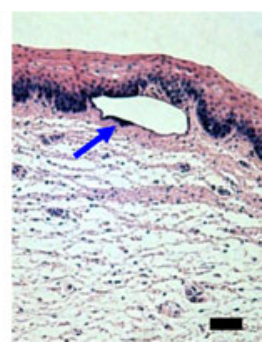

Fig. 3. Histological follow-up of voids created in porcine vocal fold with $500 \mathrm{~nJ}$ pulses. (a) Cross-sectional second harmonic generation image of a void ablated approximately $100 \mu \mathrm{m}$ beneath the tissue surface. Black arrows denote the plane targeted for ablation. (b) Follow-up histology of the void shown in (a). Red arrow indicates sub-epithelial void created by ultrafast laser ablation. (c) Histology section of a different void in the series. Blue arrow indicates small section of epithelial nuclei that were separated out of the epithelium during ultrafast laser ablation. Note the thicker void and the thinner epithelium in comparison with (b). Histology images are stained by $\mathrm{H} \& \mathrm{E}$. All scale bars are $50 \mu \mathrm{m}$. Modified from [116].

$2 \times 1 \mathrm{~mm}^{2}$ in a scarred hamster cheek pouch, as shown in Fig. 4 . In this study, $776 \mathrm{~nm}, 3 \mathrm{ps}$ pulses of $1 \mu \mathrm{J}$ energy were delivered at $300 \mathrm{kHz}$ from a fiber laser to create sub-epithelial voids $90 \mu \mathrm{m}$ below the tissue surface using a $0.75 \mathrm{NA}, 20 \times$ objective (Nikon, PlanApo). When injecting without sub-epithelial voids, we observed a back-flow of the injected biomaterial along the point of injection [Fig. 4(a)], which ultimately prevented localization of the biomaterial at the desired location. Specifically, Fig. 4(a) shows that the fluorescence observed after injection without a void is in fact originating from biomaterial that had flowed back out to cover the tissue surface, and is removed by wiping the tissue after injection. In contrast, the presence of sub-epithelial voids provides a space for the biomaterial, which greatly reduced back-flow at the injection site and resulted in a lasting localization of the injection material [Fig. 4(b)].

\section{RECENT DEVELOPMENTS TOWARD ENDOSCOPIC ULTRAFAST LASER SURGERY}

Many clinical applications require flexible delivery of the surgical laser pulses to the clinical region of interest using fiber optics. While fiber optic delivery is common practice for many conventional $\mathrm{cw}$ and long pulse lasers, fiber propagation poses several challenges for ultrafast pulses. The first challenge is that of group velocity dispersion (GVD). GVD occurs when the velocity of light exhibits a dependence on the wavelength of the light, causing the different spectral components in a single laser pulse to travel at different speeds. Shorter laser pulses necessarily contain greater spectral bandwidth so that, when all spectral components are in phase, interference among the spectral components leads to a short pulse in the time domain. The relatively large spectral bandwidth of ultrashort pulses makes them susceptible to GVD, which arises primarily from either material dispersion or waveguide dispersion. First-order linear dispersion can commonly be compensated for through the use of either prism pair or grating pair to introduce GVD of an opposite direction, in what is known as pulse chirping.

Through the optical Kerr effect and $n_{2}$, described earlier, the speed of light can also depend on the instantaneous pulse 


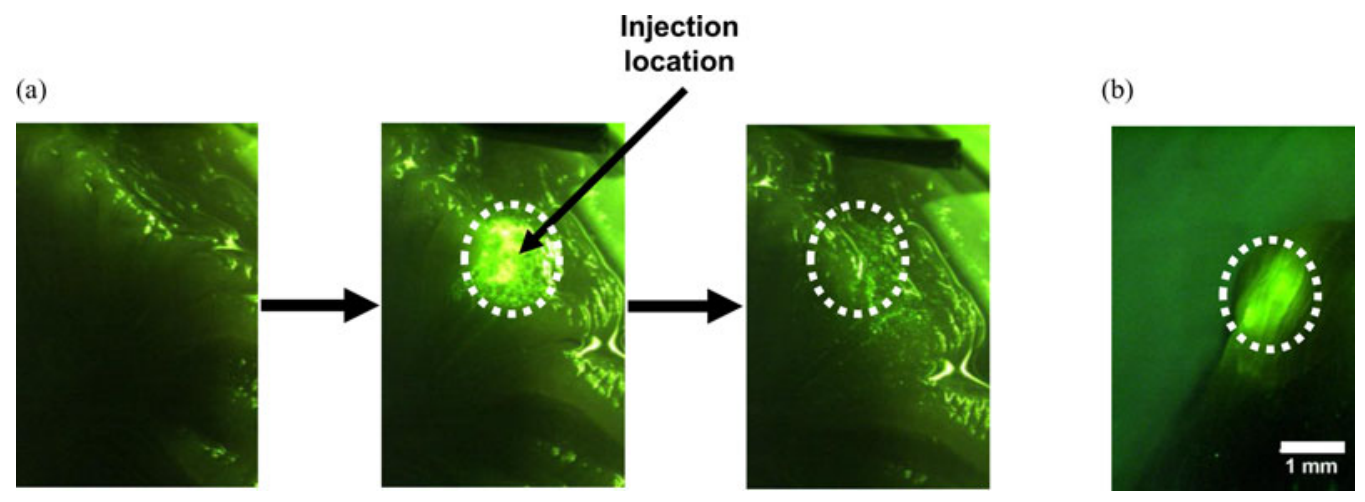

Fig. 4. Injection of PEG30 biomaterial into scarred tissue with and without ablation. Fluorescence images of (a) injection without ablation, from left to right: before injection, just after the injection, and after cleaning the tissue surface showing that it was not possible to inject and localize the biomaterial inside the scar tissue. The dashed lines indicate the region of injection. (b) Injection into an ablated void of $2 \times 1 \mathrm{~mm}^{2}$ size after wiping of tissue surface. The fluorescence signal originated from the Rhodamine dye embedded in the biomaterial. In this trial, successful localization of the biomaterial was only possible when injecting into an ablated void. Scale bars are $1 \mathrm{~mm}$.

intensity for high peak power laser pulses. In this case, the change in the local speed of light during the propagation of the pulse can create a phase shift across the pulse. This phenomenon is referred to as self-phase modulation (SPM). Because new spectral bandwidth is being created during SPM, the onset of SPM alongside GVD can result in accelerated pulse broadening in media with normal dispersion until the peak power is no longer sufficient for SPM. In this manner, SPM can lead to nonlinear dispersion properties, particularly in conventional optical fibers where GVD is nearly always present and high peak powers arise due to spatial confinement. This nonlinearity of the dispersion makes pre-compensation with a pulse chirping system extremely difficult and thus SPM must be avoided in delivering high peak power ultrashort laser pulses through normally dispersive media for microsurgery.

In the absence of SPM, such as during propagation through air-core photonic bandgap fibers, waveguide dispersion can be compensated for and ultrafast pulses capable of initiating optical breakdown in cells and tissues can be delivered [118]. In this case, the limit to the maximum deliverable peak power becomes the damage threshold of the optical fiber during coupling. This limitation can be addressed by using fibers with a larger air core or by increasing the pulse-chirping prior to the fiber to reduce the peak intensity during coupling while appropriately increasing the fiber length to restore the ultrafast pulse duration at the sample.

In addition to flexible delivery, full realization of the potential surgical precision of ultrafast laser ablation would benefit from image guidance that can resolve the features to be ablated. Nonlinear optical techniques, such as multiphoton excited fluorescence, SHG, and third harmonic generation microscopy (THG), can utilize the ultrafast microsurgical laser at reduced pulse energies to provide imaging signals. By scanning the laser beam, images of the surgical site can be created with sub-cellular details. Nonlinear imaging signals can provide both molecular and morphological information while potentially using the same microsurgical laser. As demonstrated in the vocal fold study described in Hoy et al. [116], image guidance offers crucial feedback for localization of the ablation site and for providing visual confirmation of the success and extent of ablation. In this study, the presence of increased collagen just below the epidermis acts as a natural SHG marker of the target area. Similarly, Farrar et al. have demonstrated the myelin specificity of THG [119], which could potentially guide ultrafast laser neurosurgery in the spinal cord to avoid damaging axons and make THG an ideal tool for the study of myelin loss and recovery.

Our group has been developing fiber-coupled ultrafast laser surgery probes. The first probe, shown in Fig. 5(a), consisted of a 1-m air-core photonic bandgap fiber for delivery of amplified and unamplified femtosecond laser pulses, a microelectromechanical system (MEMS) scanning mirror, a micro-optical system of aspheric and gradient index (GRIN) lenses for beam delivery and focusing. This probe also incorporated a large-core, large-NA fiber for collection of emitted photons from multiphoton fluorescence and SHG, thus enabling the use of low-energy high-repetition rate laser pulses for surgical guidance by nonlinear microscopy [118]. As mentioned above, the use of the air-core photonic crystal fiber avoids damage to the delivery fiber and nonlinear broadening of the pulse through self-phase modulation while maintaining a single-mode laser beam. By using suitable compensation for waveguide dispersion, the probe delivered $180 \mathrm{fs}$ laser pulses at $780 \mathrm{~nm}$ to the focal plane, with resulting peak irradiances in excess of $14 \mathrm{TW} / \mathrm{cm}^{2}$, well above the threshold for optical breakdown. With this first probe, precise image-guided cellular surgery was demonstrated by imaging fluorescently labeled breast carcinoma cells in cell cultures and scattering phantoms and then ablating selected single cells, shown in Fig. 5(d). While this probe demonstrated the potential for image-guided ultrafast laser surgery in a fiber-coupled probe, the dimensions of this probe would require an $18 \mathrm{~mm}$ diameter delivery channel, which precluded endoscopic delivery.

A second probe was developed based on the same architecture which reduced the probe size to less than $10 \mathrm{~mm}$ in diameter [120], as seen in Fig. 5(b). Compared to the first probe, the second probe improved the lateral and axial resolution by $20 \%$ and $40 \%$ to 1.27 and $13.5 \mu \mathrm{m}$, respectively, while reducing the diameter by almost half. Design analysis demonstrated the potential for further improvement with the use of custom lenses. 
(a) (2008)

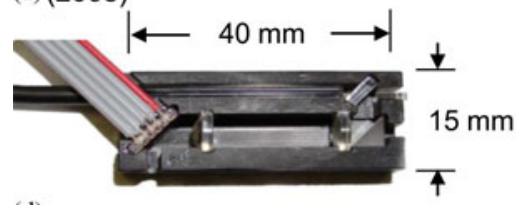

(d)

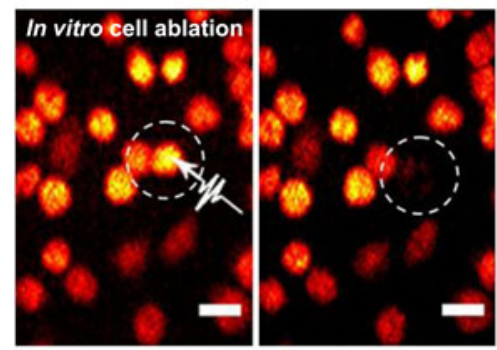

(b) (2011)

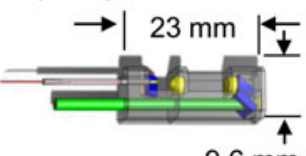

$9.6 \mathrm{~mm}$

(e)
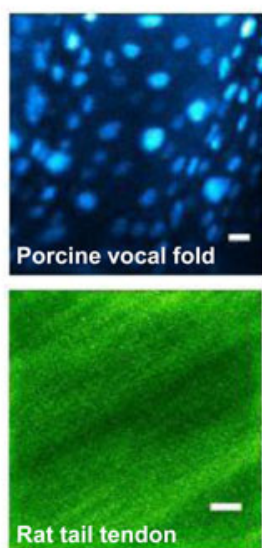

(c) $(2013)$

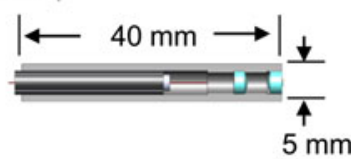

(f)

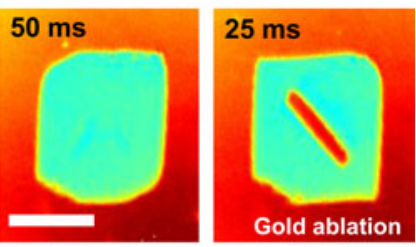

Scar ablation

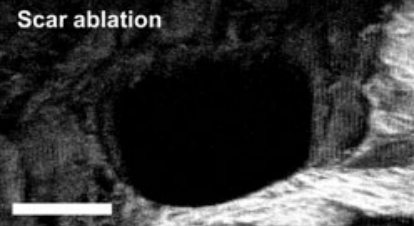

Fig. 5. Three generations of endoscopic ultrafast laser surgery probes. Photograph of the (a) 18-mm probe [118] and schematics of the (b) 9.6-mm probe [120] and the (c) 5-mm probe [121]. (d) Two-photon image of a single layer of live breast carcinoma cells after uptake of calcein AM taken prior to irradiation with high intensity pulses (left) and the same immediately after irradiation with a single pulse at $280 \mathrm{~nJ}$ pulse energy (right) using the 18 -mm probe. Scale bars are $20 \mu \mathrm{m}$. (e) (Top) A maximum intensity projection of a $\sim 70 \mu \mathrm{m}$ thick two-photon fluorescence image stack of freshly excised porcine vocal fold, stained with Hoechst 3342 , showing nuclear details, acquired with the $9.6-\mathrm{mm}$ probe. (Bottom) A SHG image of excised rat tail tendon, showing highly aligned collagen fibers. Scale bars are $10 \mu \mathrm{m}$ and $5 \mu \mathrm{m}$, respectively. (f) Ablation of a 30-nm gold coated glass slide using the 5 -mm probe, scanned for $50 \mathrm{~ms}$ (left) and $25 \mathrm{~ms}$ (right) durations in a Lissajous pattern. The entire gold within the FOV is successfully ablated at durations of $50 \mathrm{~ms}$ and above. Ultrafast laser drilling through an ex vivo $70-\mu \mathrm{m}$ thick scarred hamster cheek pouch using $200 \mathrm{~nJ}$ pulse energy (bottom); an $x y$ maximum intensity projection acquired using a benchtop nonlinear microscope visualizing SHG following ablation. Scale bars are $100 \mu \mathrm{m}$.

These improvements enabled imagining of stained tissues and of intrinsic tissue signals from SHG, as shown in Fig. 5(e) in the upper and bottom images, respectively.

Our most recently developed third probe offers a further reduction in diameter and provides an improved microsurgery speed through utilization of a compact, high repetition rate (300 kHz) erbium-doped ultrafast fiber laser $(1552 \mathrm{~nm} / 776 \mathrm{~nm}$, 3 W Discovery, Raydiance Inc.) [121]. This probe consists of a piezoelectric tube actuator for fiber scanning and two aspherical lenses that collimate and focus the light, resulting in a simple in-line optical architecture with 5-mm overall housing diameter, as shown in Fig. 5(c). In this probe, we further improved the lateral and axial resolutions, which were measured to be $1.16 \mu \mathrm{m}$ and $11.35 \mu \mathrm{m}$, respectively. A FOV of $150 \times 150 \mu \mathrm{m}$ could be scanned in a Lissajous pattern using peak voltages as low as $20 \mathrm{~V}$, complying with safety limits within human body. With the given FOV and resolution, near $100 \%$ of the resolvable spots are sampled at least once in only 50 milliseconds. Fig. 5(f) (upper) illustrates ablation of gold on glass samples as exposed to two different scanning durations. The entire FOV could be ablated for $50 \mathrm{~ms}$ scanning duration, whereas only $\sim 80 \%$ of the FOV could be ablated within 25 ms duration, leaving a small nonablated area in the middle. These results demonstrate a potential speed of microsurgery as fast as $1 \mathrm{~mm}^{2} / \mathrm{s}$ for near total ablation.

Using this 5-mm probe, we demonstrated ablation of ex vivo scarred hamster cheek pouch using $200 \mathrm{~nJ}$ pulses. We could drill through the fixed tissue, which was approximately $70 \mu \mathrm{m}$ thick, in under $10 \mathrm{~s}$. Fig. 5(f) (lower) illustrates the post-ablation SHG image of the tissue, acquired with a bench-top nonlinear microscope. Despite the narrow bandwidth of the fiber laser used in this study, this probe could still provide fluences that were higher than optical breakdown in the absence of pre-chirping to reduce laser intensities during fiber-coupling. With further development, this probe can serve as a precise and rapid ultrafast laser scalpel in the clinic.

\section{CONCLUSION}

We have attempted to provide the reader with a brief review of the mechanisms that dictate the range of applications of ultrafast lasers in surgery and a summary of the current state of clinical development. Owing to the nonlinear optical breakdown mechanism, ultrafast lasers are capable of ablating biological material with up to sub-cellular precision and can create confined, largely nonthermal cuts inside bulk tissue. While less expensive UV pulsed lasers can be utilized with similar precision for surface ablation, the ability to create precise interior cuts represents a clinical niche for ultrafast lasers. This ability has been exploited to great effect in the field of ophthalmology, where the transparent tissue of the eye and the preexisting use of laser techniques have lowered the barrier to clinical adoption.

In other areas of medicine, however, ultrafast laser surgery remains predominantly confined to the laboratory where the focus has been on investigating and developing new clinical applications. New clinical applications will likely need to identify areas where the need for precise ablation in bulk tissue justifies a potential increase in cost and complexity over existing techniques or where no other acceptable technique exists. The potential for combined surgical guidance using the same laser system for nonlinear optical imaging may also drive new applications, 
such as in neurosurgery with myelin-specific THG imaging. We have highlighted one such application, the creation of subepithelial voids for treatment of scarred vocal folds, which fits both of these criteria. Using ultrafast lasers for both surgery and image guidance, we have shown here that the ultrafast lasers can be used to greatly enhance the localization and retention of injected biomaterials used for treating vocal fold scars.

As new clinical applications are developed, ultrafast laser technology will need to develop alongside them. The size, cost, robustness, and speed of ultrafast laser systems have all improved significantly since their invention, particularly in the case of fiber lasers. Current ultrafast laser systems typically provide repetition rates in the many hundreds of kilohertz to single megahertz range, enabling faster surgical cutting and reasonable imaging times. Meanwhile, such systems deliver pulse durations frequently in the many hundreds of femtoseconds to few picoseconds, which is a desirable range for limiting the effects of self-focusing and thus ablating deeper in tissue. In addition to the lasers themselves, many applications require small and flexible delivery of the ultrafast laser light. Such delivery is possible without sacrificing the quality of the nonlinear ablation or the potential of inline image guidance through the use of photonic crystal fibers and miniaturized optical probes.

As these applications and technologies develop in the coming years, ultrafast lasers have the potential to provide many new clinical solutions and enable creative medical treatments as yet unimagined.

\section{REFERENCES}

[1] T. H. Maiman, "Stimulated optical radiation in ruby," Nature, vol. 187, pp. 493-494, 1960.

[2] C. H. Townes, "Optical masers and their possible applications to biology," Biophysical J., vol. 2, pp. 325-329, Mar. 1962.

[3] M. Bessis, F. Gires, G. Mayer, and G. Nomarski, "Irradiation des organites cellulaires a l'aide d'un LASER a rubis," Comptes Rendus de l'Academie des Sci., vol. 255, p. 1010, 1962.

[4] N. M. Saks and C. A. Roth, "Ruby laser as a microsurgical instrument," Science, vol. 141, pp. 46-47, Jul. 5, 1963.

[5] R. L. Amy and R. Storb, "Selective mitochondrial damage by a ruby laser microbeam: An electron microscope study," Science, vol. 150, pp. 756758, Nov. 5, 1965.

[6] M. W. Berns, R. S. Olson, and D. E. Rounds, "In vitro production of chromosomal lesions with an argon laser microbeam," Nature, vol. 221, pp. 74-75, 1969.

[7] M. M. Zaret, G. M. Breinin, H. Schmidt, H. Ripps, I. M. Siegel, and L. R. Solon, "Ocular lesions produced by an optical maser (laser)," Science, vol. 134, pp. 1525-1526, Nov. 10, 1961.

[8] L. Goldman, D. J. Blaney, D. J. Kindel, D. Richfield, and E. K. Franke, "Pathology of the effect of the laser beam on the skin," Nature, vol. 197, pp. 912-914, 1963.

[9] L. Goldman, P. Hornby, R. Meyer, and B. Goldman, "Impact of the laser on dental caries," Nature, vol. 203, pp. 417-417, 1964.

[10] V. Magidson, J. Loncarek, P. Hergert, C. L. Rieder, and A. Khodjakov, "Laser microsurgery in the GFP Era: A cell biologist's perspective," Methods Cell Biol., vol. 82, pp. 239-266, Jun. 21, 2007.

[11] F. Fankhauser and S. Kwasniewska, Lasers in Ophthalmology_Basic, Diagnostic, and Surgical Aspects: A Review. The Hague, The Netherlands: Kugler, 2003.

[12] E. L. Tanzi, J. R. Lupton, and T. S. Alster, "Lasers in dermatology: Four decades of progress," J. Amer. Academy Dermatol., vol. 49, pp. 1-31, 2003.

[13] R. H. Ossoff, J. A. Coleman, M. S. Courey, J. A. Duncavage, J. A. Werkhaven, and L. Reinisch, "Clinical applications of lasers in otolaryngology - Head and neck surgery," Lasers Surg. Med., vol. 15, pp. 217-248, 1994.

[14] A. Vogel and V. Venugopalan, "Mechanisms of pulsed laser ablation of biological tissues," Chem. Rev., vol. 103, pp. 577-644, 2003.
[15] L. S. Bass and M. R. Treat, "Laser tissue welding: A comprehensive review of current and future," Lasers Surg. Med., vol. 17, pp. 315-349, 1995.

[16] A. P. Joglekar, H. H. Liu, E. Meyhofer, G. Mourou, and A. J. Hunt, "Optics at critical intensity: Applications to nanomorphing," Proc. Nat. Academy Sci. USA, vol. 101, pp. 5856-5861, Apr. 20, 2004.

[17] A. Vogel, J. Noack, G. Hüttman, and G. Paltauf, "Mechanisms of femtosecond laser nanosurgery of cells and tissues," Appl. Phys. B, vol. 81, pp. 1015-1047, Dec. 2005 .

[18] B. C. Stuart, M. D. Feit, A. M. Rubenchik, B. W. Shore, and M. D. Perry, "Laser-induced damage in dielectrics with nanosecond to subpicosecond pulses," Phys. Rev. Lett., vol. 74, pp. 2248-2251, Mar. 20, 1995.

[19] U. K. Tirlapur, K. König, C. Peuckert, R. Krieg, and K.-J. Halbhuber "Femtosecond near-infrared laser pulses elicit generation of reactive oxygen species in mammalian cells leading to apoptosis-like death," Exp. Cell Res., vol. 263, pp. 88-97, Jan. 3, 2001.

[20] B. Boudaiffa, P. Cloutier, D. Hunting, M. A. Huels, and L. Sanche, "Resonant formation of DNA strand breaks by low-energy (3- to 20- eV) Electrons," Science, vol. 287, pp. 1658-1660, Mar. 2000.

[21] L. R. Koller, Ultraviolet Radiation, 2nd ed. New York, NY, USA: Wiley, 1965.

[22] D. Ashkenasi, M. Lorenz, R. Stoian, and A. Rosenfeld, "Surface damage threshold and structuring of dielectrics using femtosecond laser pulses: The role of incubation," Appl. Surface Sci., vol. 150, pp. 101-106, 1999.

[23] F. Bourgeois and A. Ben-Yakar, "Femtosecond laser nanoaxotomy properties and their effect on axonal recovery in C. Elegans," Opt. Exp., vol. 15, pp. 8521-8531, Jun. 25, 2007.

[24] A. Ben-Yakar and F. Bourgeois, "Ultrafast laser nanosurgery in microfluidics for genome-wide screenings," Current Opinion Biotechnol., vol. 20, pp. 100-105, 2009.

[25] G. Olivié, D. Giguère, F. Vidal, T. Ozaki, J. C. Kieffer, O. Nada, and I. Brunette, "Wavelength dependence of femtosecond laser ablation threshold of corneal stroma," Opt. Exp., vol. 16, pp. 4121-4129, 2008.

[26] R. Y. Chiao, E. Garmire, and C. H. Townes, "Self-Trapping of optical beams," Phys. Rev. Lett., vol. 13, pp. 479-482, 1964.

[27] P. L. Kelley, "Self-focusing of optical beams," Phys. Rev. Lett., vol. 15 , pp. 1005-1008, 1965.

[28] E. T. J. Nibbering, M. A. Franco, B. S. Prade, G. Grillon, C. Le Blanc, and A. Mysyrowicz, "Measurement of the nonlinear refractive index of transparent materials by spectral analysis after nonlinear propagation," Opt. Commun., vol. 119, pp. 479-484, 1995.

[29] M. Miclea, U. Skrzypczak, S. Faust, F. Fankhauser, H. Graener, and G. Seifert, "Nonlinear refractive index of porcine cornea studied by $\mathrm{z}$ scan and self-focusing during femtosecond laser processing," Opt. Exp., vol. 18, pp. 3700-3707, 2010.

[30] Y. R. Shen, The Principles of Nonlinear Optics. New York, NY, USA Wiley, 1984.

[31] C. L. Arnold, A. Heisterkamp, W. Ertmer, and H. Lubatschowski, "Streak formation as side effect of optical breakdown during processing the bulk of transparent Kerr media with ultra-short laser pulses," Appl. Phys. B, Lasers Opt., vol. 80, pp. 247-253, 2005.

[32] A. Heisterkamp, T. Ripken, T. Mamom, W. Drommer, H. Welling, W. Ertmer, and H. Lubatschowski, "Nonlinear side effects of fs pulses inside corneal tissue during photodisruption," Appl. Phys. B-Lasers Opt., vol. 74, pp. 419-425, Apr. 2002.

[33] M. Han, L. Zickler, M. Walter, G. Giese, F. Loesel, and J. F. Bille, "Noninvasive evaluation of mini-invasive femtosecond laser refractive surgery," in Proc. Commercial Biomed. Appl. Ultrafast Lasers IV, San Jose, CA, USA, 2004, pp. 55-65.

[34] L. Sudrie, A. Couairon, M. Franco, B. Lamouroux, B. Prade, S. Tzortzakis, and A. Mysyrowicz, "Femtosecond laser-induced damage and filamentary propagation in fused silica," Phys. Rev. Lett., vol. 89, pp. 186601-1-186601-4, 2002.

[35] Tse, Christine, Zohdy, J. Marwa, Y. E. Jing Yong, O'Donnell, and Matthew, "Penetration and precision of subsurface photodisruption in porcine skin tissue with infrared femtosecond laser pulses," IEEE Trans. Biomed. Eng., vol. 55, no. 3, pp. 1211-1218, Mar. 2008.

[36] R. Meesat, H. Belmouaddine, J.-F. O. Allard, C. Tanguay-Renaud, R. Lemay, T. Brastaviceanu, L. Tremblay, B. Paquette, J. R. Wagner, J.-P. Jay-Gerin, M. Lepage, M. A. Huels, and D. Houde, "Cancer radiotherapy based on femtosecond IR laser-beam filamentation yielding ultra-high dose rates and zero entrance dose," Proc. Nat. Academy Sci. USA, vol. 109, pp. E2508-E2513, Sep. 18, 2012.

[37] C. B. Schaffer, A. Brodeur, J. F. García, and E. Mazur, "Micromachining bulk glass by use of femtosecond laser pulses with nanojoule energy," Opt. Lett., vol. 26, pp. 93-95, 2001. 
[38] V. Nuzzo, M. Savoldelli, J. M. Legeais, and K. Plamann, "Self-focusing and spherical aberrations in corneal tissue during photodisruption by femtosecond laser," J. Biomed. Opt., vol. 15, p. 038003, May/Jun. 2010.

[39] D. Eversole, B. Luk'yanchuk, and A. Ben-Yakar, "Plasmonic laser nanoablation of silicon by the scattering of femtosecond pulses near gold nanospheres," Appl. Phys. A, vol. 89, pp. 283-291, 2007.

[40] R. K. Harrison and A. Ben-Yakar, "Role of near-field enhancement in plasmonic laser nanoablation using gold nanorods on a silicon substrate," Opt. Exp., vol. 18, pp. 22556-22571, 2010.

[41] A. Ben-Yakar et al., "Spherical and Anisotropic gold nanoparticles in plasmonic laser phototherapy of cancer," in Non-Magnetic Metallic Nanomaterials for Life Sciences (Nanomaterials for Life Sciences vol. 4), Challa Kumar, Ed., Weinheim: John \& Wiley Sons, 2008, pp. 493-539.

[42] M. E. Durst, G. Zhu, and C. Xu, "Simultaneous spatial and temporal focusing in nonlinear microscopy," Opt. Commun., vol. 281, pp. 1796$1805,2008$.

[43] R. Kammel, R. Ackermann, A. Tünnermann, and S. Nolte, "Pump-probe investigation of fs-LIOB in water by simultaneous spatial and temporal focusing," in Proc. SPIE, Mar. 15, 2013, vol. 8611, p. 86110A.

[44] F. He, H. Xu, Y. Cheng, J. Ni, H. Xiong, Z. Xu, K. Sugioka, and K. Midorikawa, "Fabrication of microfluidic channels with a circular cross section using spatiotemporally focused femtosecond laser pulses," Opt. Lett., vol. 35, pp. 1106-1108, 2010.

[45] D. N. Vitek, D. E. Adams, A. Johnson, P. S. Tsai, S. Backus, C. G. Durfee, D. Kleinfeld, and J. A. Squier, "Temporally focused femtosecond laser pulses for low numerical aperture micromachining through optically transparent materials," Opt. Exp., vol. 18, pp. 1808618094,2010

[46] E. Block, M. Greco, D. Vitek, O. Masihzadeh, D. A. Ammar, M. Y. Kahook, N. Mandava, C. Durfee, and J. Squier, "Simultaneous spatial and temporal focusing for tissue ablation," Biomed. Opt. Exp., vol. 4, pp. 831-841, 2013.

[47] D. Stern, R. W. Schoenlein, C. A. Puliafito, E. T. Dobi, R. Birngruber, and J. G. Fujimoto, "Corneal ablation by nanosecond, picosecond, and femtosecond lasers at 532 and $625 \mathrm{Nm}$," Archives Ophthalmol., vol. 107 pp. 587-592, Apr. 1989

[48] W. Kautek, S. Mitterer, J. Krüger, W. Husinsky, and G. Grabner, "Femtosecond-pulse laser ablation of human corneas," Appl. Phys. A, Mater. Sci. Process., vol. 58, pp. 513-518, 1994.

[49] F. H. Loesel, J. P. Fischer, M. H. Gotz, C. Horvath, T. Juhasz, F. Noack, N. Suhm, and J. F. Bille, "Non-thermal ablation of neural tissue with femtosecond laser pulses," Appl. Phys. B, Lasers Opt., vol. 66, pp. 121128, Jan. 1998.

[50] J. Neev, L. B. D. Silva, M. D. Feit, M. D. Perry, A. M. Rubenchik, and B. C. Stuart, "Ultrashort pulse lasers for hard tissue ablation," IEEE J. Sel. Topics Quantum Electron., vol. 2, no. 4, pp. 790-800, Dec. 1996.

[51] I. Ratkay-Traub, I. E. Ferincz, T. Juhasz, R. M. Kurtz, and R. R. Krueger, "First clinical results with the femtosecond neodynium-glass laser in refractive surgery," J. Refract. Surg., vol. 19, pp. 94-103, Mar./Apr. 2003.

[52] K. Stonecipher, T. S. Ignacio, and M. Stonecipher, "Advances in refractive surgery: Microkeratome and femtosecond laser flap creation in relation to safety, efficacy, predictability, and biomechanical stability," Current Opinion Ophthalmol., vol. 17, pp. 368-372, 2006.

[53] S. G. Slade, "The use of the femtosecond laser in the customization of corneal flaps in laser in situ keratomileusis," Current Opinion Ophthalmol., vol. 18, pp. 314-317, Jul. 2007.

[54] H. Lubatschowski, "Overview of commercially available femtosecond lasers in refractive surgery," J. Refract. Surg., vol. 24, pp. 102-107, Jan. 2008.

[55] S. G. Slade, "Applications for the femtosecond laser in corneal surgery," Current Opinion Ophthalmol., vol. 18, pp. 338-341, Jul. 2007.

[56] B. Seitz, A. Langenbucher, C. Hofmann-Rummelt, U. SchlötzerSchrehardt, and G. O. H. Naumann, "Nonmechanical posterior lamellar keratoplasty using the femtosecond laser (femto-plak) for corneal endothelial decompensation," Amer. J. Ophthalmol., vol. 136, pp. 769772, 2003.

[57] S. I. M. D. Mian, H. K. M. D. Soong, S. V. M. D. Patel, T. M. D. Ignacio, and T. P. Juhasz, "In vivo femtosecond laser-assisted posterior lamellar keratoplasty in rabbits," Cornea, vol. 25, pp. 1205-1209, 2006.

[58] L. Buratto and E. Böhm, "The use of the femtosecond laser in penetrating keratoplasty," Amer. J. Ophthalmol., vol. 143, pp. 737-742, 2007.

[59] T. S. Ignacio, T. B. Nguyen, R. S. Chuck, R. M. Kurtz, and M. A. Sarayba, "Top hat wound configuration for penetrating keratoplasty using the femtosecond laser: A laboratory model," Cornea, vol. 25, pp. 336-340, 2006.
[60] M. Farid, M. Kim, and R. F. Steinert, "Results of penetrating keratoplasty performed with a femtosecond laser zigzag incision initial report," Ophthalmology, vol. 114, pp. 2208-2212, 2007.

[61] F. W. Price, Jr. and M. O. Price, "Femtosecond laser shaped penetrating keratoplasty: One-year results utilizing a top-hat configuration," Amer. J. Ophthalmol., vol. 145, pp. 210-214, 2008.

[62] H. K. Soong and J. B. Malta, "Femtosecond lasers in ophthalmology," Amer. J. Ophthalmol., vol. 147, pp. 189-197, Feb. 2009.

[63] Z. Nagy, A. Takacs, T. Filkorn, and M. Sarayba, "Initial clinical evaluation of an intraocular femtosecond laser in cataract surgery," J. Refract. Surg., vol. 25, pp. 1053-1060, 2009.

[64] D. V. Palanker, M. S. Blumenkranz, D. Andersen, M. Wiltberger, G. Marcellino, P. Gooding, D. Angeley, G. Schuele, B. Woodley, M. Simoneau, N. J. Friedman, B. Seibel, J. Batlle, R. Feliz, J. Talamo, and W. Culbertson, "Femtosecond laser-assisted cataract surgery with integrated optical coherence tomography," Sci. Transl. Med., vol. 2, pp. 58ra85-1-58ra85-9, Nov. 17, 2010.

[65] K. Kránitz, A. Takacs, K. Miháltz, I. Kovács, M. C. Knorz, and Z. Z. Nagy, "Femtosecond laser capsulotomy and manual continuous curvilinear capsulorrhexis parameters and their effects on intraocular lens centration," J. Refract. Surg., vol. 27, pp. 558-563, 2011.

[66] N. J. Friedman, D. V. Palanker, G. Schuele, D. Andersen, G. Marcellino, B. S. Seibel, J. Batlle, R. Feliz, J. H. Talamo, M. S. Blumenkranz, and W. W. Culbertson, "Femtosecond laser capsulotomy," J. Cataract Refract. Surg., vol. 37, pp. 1189-1198, 2011.

[67] Y. S. Rabinowitz, X. Li, T. S. Ignacio, and E. Maguen, "INTACS inserts using the femtosecond laser compared to the mechanical spreader in the treatment of keratoconus," J. Refract. Surg., vol. 22, pp. 764-771, 2006.

[68] A. Ertan, G. Kamburoglu, and M. Bahadir, "Intacs insertion with the femtosecond laser for the management of keratoconus-One-year results," J. Cataract Refract. Surg., vol. 32, pp. 2039-2042, Dec. 2006.

[69] M. H. Shabayek and J. L. Alió, "Intrastromal corneal ring segment implantation by femtosecond laser for keratoconus correction," Ophthalmology, vol. 114, pp. 1643-1652, Sep. 2007.

[70] E. Coskunseven, G. D. Kymionis, N. S. Tsiklis, S. Atun, E. Arslan, M. R. Jankov, and I. G. Pallikaris, "One-year results of intrastromal corneal ring segment implantation (keraring) using femtosecond laser in patients with keratoconus," Amer. J. Ophthalmol., vol. 145, pp. 775779, May 2008

[71] S. Yoo, G. Kymionis, T. O'Brien, T. Ide, W. Culbertson, and E. Alfonso, "Femtosecond-assisted diagnostic corneal biopsy (FAB) in keratitis," Graefe's Archive Clin. Exp. Ophthalmol., vol. 246, pp. 759-762, 2008.

[72] J. H. Kim, J. H. Yum, D. Lee, and S. H. Oh, "Novel technique of corneal biopsy by using a femtosecond laser in infectious ulcers," Cornea, vol. 27, pp. 363-365, Apr. 2008.

[73] H. Louis, P. Helene, M. Frederic, C. John, and R. Bernard, "Correction of postkeratoplasty astigmatism by femtosecond laser compared with mechanized astigmatic keratotomy," Amer. J. Ophthalmol., vol. 147, pp. 779-787, May 2009.

[74] M. Blum, K. Kunert, S. Nolte, S. Riehemann, M. Palme, T. Peschel, M. Dick, and H. Dick, "Presbyopietherapie mit Femtosekundenlaser," Der Ophthalmol., vol. 103, pp. 1014-1019, 2006.

[75] T. Ripken, U. Oberheide, M. Fromm, S. Schumacher, G. Gerten, and H. Lubatschowski, "fs-Laser induced elasticity changes to improve presbyopic lens accommodation," Graefe's Archive Clin. Exp. Ophthalmol., vol. 246, pp. 897-906, 2008.

[76] S. Schumacher, M. Fromm, U. Oberheide, G. Gerten, A. Wegener, and H. Lubatschowski, "In vivo application and imaging of intralenticular femtosecond laser pulses for the restoration of accommodation," J. Refract. Surg., vol. 24, pp. 991-995, Nov. 2008.

[77] S. Schumacher, U. Oberheide, M. Fromm, T. Ripken, W. Ertmer, G. Gerten, A. Wegener, and H. Lubatschowski, "Femtosecond laser induced flexibility change of human donor lenses," Vis. Res., vol. 49, pp. 1853-1859, Jul. 2009.

[78] M. A. Sarayba, R. M. Kurtz, T. T. B. Nguyen, T. Ignacio, M. Mansoori, P. M. Sweet, and R. S. Chuck, "Femtosecond laser-assisted intracorneal keratoprosthesis implantation-A laboratory model," Cornea, vol. 24, pp. 1010-1014, Nov. 2005.

[79] K. S. Frederickson, W. E. White, R. G. Wheeland, and D. R. Slaughter, "Precise ablation of skin with reduced collateral damage using the femtosecond-pulsed, terawatt titanium-sapphire laser," Arch Dermatol., vol. 129, pp. 989-993, Aug. 1, 1993.

[80] N. Nishimura, C. B. Schaffer, E. H. Li, and E. Mazur, "Tissue ablation with 100-fs and 200-ps laser pulses," in Proc. IEEE 20th Annu. Int. Conf. Eng. Med. Biol. Soc., 1998, vol. 20, pp. 1703-1706. 
[81] H. Wisweh, U. Merkel, A. K. Huller, K. Lurben, and H. Lubatschowski, "Optical coherence tomography monitoring of vocal fold femtosecond laser microsurgery," in Therapeutic Laser Applications and Laser-Tissue Interaction III, 2007, p. 63207.

[82] H. Huang and Z. Guo, "Human dermis separation via ultra-short pulsed laser plasma-mediated ablation," J. Phys. D, Appl. Phys., vol. 42, pp. 165204-1-165204-9, 2009.

[83] H. Huang and Z. Guo, "Ultrashort pulsed laser ablation and stripping of freeze-dried dermis," Lasers Med. Sci., vol. 25, pp. 517-524, 2010.

[84] H. F. Loesel, H. M. Niemz, F. J. Bille, and T. Juhasz, "Laser-induced optical breakdown on hard and soft tissues and its dependence on the pulse duration: Experiment and model,". (1996, Oct.). IEEE J. Quantum Electron., vol. 32, no. 10, pp. 1717-1722, 1996.

[85] J. Wahrburg, K.-U. Schmidt, M. H. Götz, K. Kappings, and S. Gölz, "Concept of a novel laser probe for minimal invasive applications in neurosurgery," Mechatronics, vol. 6, pp. 479-489, 1996.

[86] M. H. Goetz et al., "Computer-guided laser probe for ablation of brain tumours with ultrashort laser pulses," Phys. Med. Biol., vol. 44, pp. N119127, 1999.

[87] J. Nguyen, J. Ferdman, M. Zhou, D. Huland, S. Saqqa, J. Ma, N. Nishimura, T. Schwartz, and C. Schaffer, "Femtosecond laser ablation as a subsurface light scalpel to alter seizure propagation dynamics," presented at Amer. Soc. Lasers Med. Surg. Annu. Meeting, Phoenix, AZ, USA, 2010.

[88] A. Moritz and F. Beer, Oral Laser Application. London, U.K.: Quintessence, 2006.

[89] J. Neev, L.-H. L. Liaw, D. V. Raney, J. T. Fujishige, P. D. Ho, and M. W. Berns, "Selectivity, efficiency, and surface characteristics of hard dental tissues ablated with ArF pulsed excimer lasers," Lasers Surg. Med., vol. 11, pp. 499-510, 1991.

[90] J. Pelagalli, C. B. Gimbel, R. T. Hansen, A. Swett, and D. W. Winn, "Investigational study of the use of Er:YAG laser versus dental drill for caries removal and cavity preparation-Phase I," J. Clin. Laser Med. Surg., vol. 15, pp. 109-115, 1997.

[91] L. Zach and G. Cohen, "Pulp response to externally applied heat," Oral Surg., Oral Med., Oral Pathol., vol. 19, pp. 515-530, 1965.

[92] K. Ulrich and H. Raimund, "Experimental studies of the application of the Er:YAG laser on dental hard substances-Part II: Light microscopic and SEM investigations," Lasers Surg. Med., vol. 9, pp. 345-351, 1989.

[93] B. Gokce, B. Ozpinar, C. Artunc, and G. Aksoy, "Laser use vs. handpiece for tooth preparation: A preliminary in vitro study," J. Laser Appl., vol. 21, pp. 63-66, 2009.

[94] O. Feuerstein, D. Palanker, A. Fuxbrunner, A. Lewis, and D. Deutsch, "Effect of the ArF excimer laser on human enamel," Lasers Surg. Med., vol. 12, pp. 471-477, 1992.

[95] R. Boehm, J. Rich, J. Webster, and S. Janke, "Thermal stress effects and surface cracking associated with laser use on human teeth," J. Biomech. Eng., vol. 99, pp. 189-194, 1977.

[96] H. H. K. Xu, J. R. Kelly, S. Jahanmir, V. P. Thompson, and E. D. Rekow, "Enamel subsurface damage due to tooth preparation with diamonds," $J$. Dental Res., vol. 76, pp. 1698-1706, Oct. 1997.

[97] B.-M. Kim, M. D. Feit, A. M. Rubenchik, E. J. Joslin, P. M. Celliers, J. Eichler, and L. B. Da Silva, "Influence of pulse duration on ultrashort laser pulse ablation of biological tissues," J. Biomed. Opt., vol. 6, pp. 332338, 2001

[98] A. V. Rode, E. G. Gamaly, B. Luther-Davies, B. T. Taylor, J. Dawes, A. Chan, R. M. Lowe, and P. Hannaford, "Subpicosecond laser ablation of dental enamel," J. Appl. Phys., vol. 92, pp. 2153-2158, 2002.

[99] J. Krüger, W. Kautek, and H. Newesely, "Femtosecond-pulse laser ablation of dental hydroxyapatite and single-crystalline fluoroapatite," Appl. Phys. A, Mater. Sci. Process., vol. 69, pp. S403-S407, 1999.

[100] M. H. Niemz, A. Kasenbacher, M. Strassl, A. Bäcker, A. Beyertt, D. Nickel, and A. Giesen, "Tooth ablation using a CPA-free thin disk femtosecond laser system," Appl. Phys. B, Lasers Opt., vol. 79, pp. 269271,2004

[101] J. Neev, J. S. Nelson, M. Critelli, J. L. McCullough, E. Cheung, W. A. Carrasco, A. M. Rubenchik, L. B. D. Silva, M. D. Perry, and B. C. Stuart, "Ablation of human nail by pulsed lasers," Lasers Surg. Med, vol. 21, pp. 186-192, 1997.

[102] J. Serbin, T. Bauer, C. Fallnich, A. Kasenbacher, and W. H. Arnold, "Femtosecond lasers as novel tool in dental surgery," Appl. Surface Sci., vol. 197, pp. 737-740, Sep. 30, 2002.

[103] L. R. Friesen, C. M. Cobb, J. W. Rapley, L. Forgas-Brockman, and P. Spencer, "Laser irradiation of bone-Part II: Healing response following treatment by CO2 and Nd:YAG lasers," J. Periodontol., vol. 70, pp. 75-83, 1999.
[104] E. K. Gardner, J. Dornhoffer, and S. Ferguson, "Photoacoustic effects of carbon dioxide lasers in stapes surgery: Quantification in a temporal bone model," Otol Neurotol., vol. 23, pp. 862-865, 2002.

[105] B. Girard, D. Yu, M. R. Armstrong, B. C. Wilson, C. M. L. Clokie, and R. J. D. Miller, "Effects of femtosecond laser irradiation on osseous tissues," Lasers Surg. Med., vol. 39, pp. 273-285, 2007.

[106] K. Ozono and M. Obara, "Tailored ablation processing of advanced biomedical hydroxyapatite by femtosecond laser pulses," Appl. Phys. A Mater. Sci. Process., vol. 77, pp. 303-306, 2003.

[107] B. Girard, M. Cloutier, D. J. Wilson, C. M. L. Clokie, R. J. D. Miller, and B. C. Wilson, "Microtomographic analysis of healing of femtosecond laser bone calvarial wounds compared to mechanical instruments in mice with and without application of BMP-7," Lasers Surg. Med., vol. 39, pp. 458-467, 2007.

[108] W. B. Armstrong, J. A. Neev, L. B. D. Silva, A. M. Rubenchik, and B. C. Stuart, "Ultrashort pulse laser ossicular ablation and stapedotomy in cadaveric bone," Lasers Surg. Med., vol. 30, pp. 216-220, 2002.

[109] J. Ilgner, M. Wehner, J. Lorenzen, M. Bovi, and M. Westhofen, "Morphological effects of nanosecond- and femtosecond-pulsed laser ablation on human middle ear ossicles," J. Biomed. Opt., vol. 11, pp. 014004-1014004-7, Jan./Feb. 2006.

[110] R. G. McCaughey, H. Sun, V. S. Rothholtz, T. Juhasz, and B. J. F. Wong, "Femtosecond laser ablation of the stapes," J. Biomed. Opt., vol. 14, pp. 024040-024046, 2009.

[111] B. J. F. Wong, M. R. Dickinson, M. W. Berns, and J. Neev, "Identification of photoacoustic transients during pulsed laser ablation of the human temporal bone: An experimental model," J. Clin. Laser Med. Surg., vol. 14, pp. 385-392, 1996.

[112] Y. Liu and M. Niemz, "Ablation of femural bone with femtosecond laser pulses-A feasibility study," Lasers Med. Sci., vol. 22, pp. 171-174, 2007.

[113] J. Qiu, J. M. H. Teichman, T. Wang, J. Neev, R. D. Glickman, K. F. Chan, and T. E. Milner, "Femtosecond laser lithotripsy: Feasibility and ablation mechanism," J. Biomed. Opt., vol. 15, pp. 028001-028005, 2010.

[114] J. Qiu, J. Neev, T. Wang, D. Gomez, and T. E. Milner, "Fiber delivery system for ablation of hard and soft tissues using near-infrared femtosecond laser pulses," in Proc. Amer. Soc. Lasers Med. Surg. Annu. Conf., Phoenix, AZ, USA, 2010.

[115] Y. Matsuura, M. Miyagi, K. Shihoyama, and M. Kawachi, "Delivery of femtosecond pulses by flexible hollow fibers," J. Appl. Phys., vol. 91, pp. 887-889, 2002.

[116] C. L. Hoy, W. N. Everett, M. Yildirim, J. Kobler, S. M. Zeitels, and A. Ben-Yakar, "Towards endoscopic ultrafast laser microsurgery of vocal folds," J. Biomed. Opt., vol. 17, pp. 038002-038001, Mar. 2012.

[117] S. Hirano, "Current treatment of vocal fold scarring," Current Opinion Otolaryngol. Head Neck Surg., vol. 13, pp. 143-147, 2005.

[118] C. L. Hoy, N. J. Durr, P. Chen, W. Piyawattanametha, H. Ra, O. Solgaard, and A. Ben-Yakar, "Miniaturized probe for femtosecond laser microsurgery and two-photon imaging," Opt. Exp., vol. 16, pp. 9996-10005, 2008

[119] M. Farrar, F. Wise, J. Fetcho, and C. Schaffer, "In vivo imaging of myelin in the vertebrate central nervous system using third harmonic generation microscopy," Biophys. J., vol. 100, pp. 1362-1371, 2011.

[120] C. L. Hoy, O. Ferhanoğlu, M. Yildirim, W. Piyawattanametha, H. Ra, O. Solgaard, and A. Ben-Yakar, "Optical design and imaging performance testing of a 9.6- $\mathrm{mm}$ diameter femtosecond laser microsurgery probe," Opt. Exp., vol. 19, pp. 10536-10552, 2011.

[121] O. Ferhanoglu, M. Yildirim, K. Subramanian, and A. Ben-Yakar, "A 5-mm piezo-actuated fiber probe for high speed ultrafast laser microsurgery," in preparation.

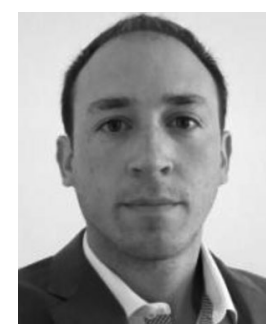

Christopher L. Hoy received the B.Sc. (cum laude) degree in mechanical engineering from Virginia Polytechnic Institute and State University (Virginia Tech), Blacksburg, VA, USA, in 2004. He received the M.Sc. and $\mathrm{Ph} . \mathrm{D}$. degrees mechanical engineering from The University of Texas at Austin, Austin, TX, USA, in 2007 and 2011, respectively, while developing femtosecond laser endoscopic microsurgery. In 2011, he joined the Center for Optical Diagnostics and Therapy at Erasmus Medical Center in Rotterdam, the Netherlands, as a postdoctoral research fellow. His current research interests include biomedical applications of ultrafast lasers for both imaging and surgery in addition to quantitative reflectance and fluorescence spectroscopy. He is a member of SPIE and OSA. 


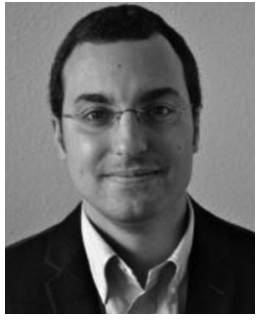

Onur Ferhanoğlu received the B.Sc. and M.Sc. degrees in electrical engineering from Bilkent University, Ankara, Turkey, in 2003 and 2005, respectively. In 2005, he joined Optical Microsystems Laboratory, Koç University as a Graduate Researcher, where he developed MEMS-based thermal imaging sensor arrays. During graduate studies, he visited Johns Hopkins University in 2004, Georgia Institute of Technology in 2007, and EPFL in 2010 as a Visiting Scientist. After receiving the Ph.D. degree in 2011, he joined Femtosecond Laser Assisted Biophotonics Laboratory at the University of Texas at Austin as a Postdoctoral Scholar. His current research interests include biomedical optics, optical interventions, and MEMS. He is a member of SPIE.

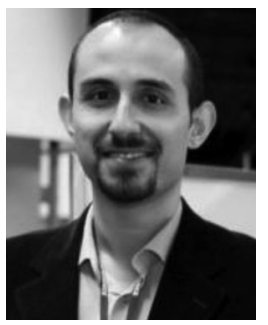

Murat Yildirim received the B.Sc. and M.Sc. degrees in mechanical engineering from Middle Eas Technical University, Turkey, in 2006 and 2009, respectively. In 2010, he joined the University of Texas at Austin as a Graduate Researcher. He was the runner-up for the JenLab Young Investigator Award for the best paper in 2013. His current research interests include nonlinear microscopy and developing miniaturized probes for imaging and surgery. He is a member of SPIE and OSA.



Ki Hyun Kim received the B.Sc. degree in physics and the B.Sc. degree in electrical engineering from Yonsei University, Seoul, Korea in 2009. He received the M.Sc. degree from the University of Texas at Austin, Austin, TX, USA, in 2011. He is currently a Graduate Student in the Department of Electrical and Computer Engineering, University of Texas at Austin. His current research interests include optical scanning and imaging systems for high throughput automated drug screening and nerve regeneration verification.

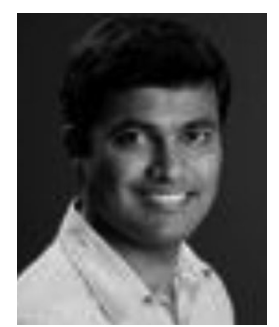

Sandeep S. Karajanagi received the B.S. degree in chemical engineering from the University of Mumbai, India, in 2001 and the Ph.D. degree from Rensselaer Polytechnic Institute in Chemical and $\mathrm{Bi}-$ ological Engineering, in 2006. He completed his postdoctoral work at the Massachusetts Institute of Technology in the Robert Langer group in chemical engineering in 2008. He is currently an Instructor of Surgery at the Harvard Medical School and the Massachusetts General Hospital. His work is focused on research and development of biomaterials and drug delivery-based technologies for biomedical problems, focused on laryngeal surgery. He is a member of the ACS, AIChE, and is the recipient of the BroylesMaloney Award given by the American Bronchoesophagological Association.

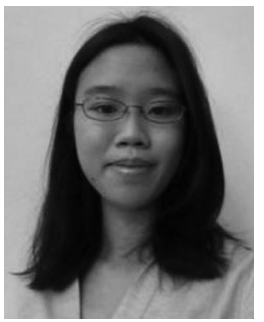

Ka Man Carmen Chan received the B.Sc. degree in chemical-biological engineering from Massachusetts Institute of Technology, Cambridge, MA, USA, in 2012. In 2009, she joined the Center for Laryngeal Surgery and Voice Rehabilitation at Massachusetts General Hospital in Boston, MA, USA, as an undergraduate research student and as a research technician in 2012. Her current research interests include developing biomaterials for clinical applications.

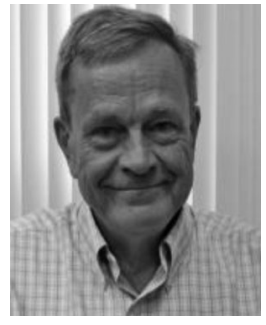

James B. Kobler received the B.A. degree (cum laude) from Vassar College, Poughkeepsie, NY, USA, in 1975 and the Ph.D. degree in neurobiology from the University of North Carolina, Chapel Hill, NC, USA, in 1983. He did postdoctoral work at the Eaton Peabody Laboratory, Massachusetts Eye and Ear Infirmary through the MIT Research Laboratory of Electronics. He is currently an Associate Physiologist at the Center for Laryngeal Surgery and Voice Rehabilitation at Massachusetts General Hospital, MA, USA, and an Assistant Professor of Surgery at Harvard Medical School, Boston, MA. His research interests include laryngeal anatomy and physiology, surgical instrumentation, surgical applications of lasers and imaging vocal fold motion with optical coherence tomography.

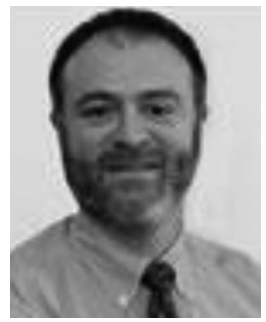

Steven M. Zeitels is the Eugene B. Casey Professor of Laryngeal Surgery at Harvard and the Director of the MGH Center for Laryngeal Surgery and Voice Rehabilitation. He has contributed more than 200 publications and videos, more than 300 papers and lectures and has been a Visiting Professor at more than 30 institutions, received more than 50 awards and honored lectureships including the Casselberry Award of the American Laryngological Association. His work has been featured in various journals and magazines, and extensively covered in the print, radio, and television media. He conceived and directs the Voice Restoration Research Program, which is a collaborative effort of investigators at HMS, MGH, MIT, and Julie Andrews. He has served as the President of the American Broncho-Esophagological Association. In 2004, the first endowed Chair in Laryngeal Surgery at Harvard Medical was created for him while he re-established a Laryngeal Surgery service at the MGH, which had not been present since the 1920s. He was a member of the 1st class of BU Trustee Scholars, attended the BU 6-year medical program and graduated the BU Medical School in 1982. He completed the BU-Tufts Otolaryngology - Head and Neck Surgery Residency in 1987 and a Head and Neck Cancer Fellowship at BU and VAMC in 1988. He received the Distinguished Alumnus Award from the BU School of Medicine in 2007.

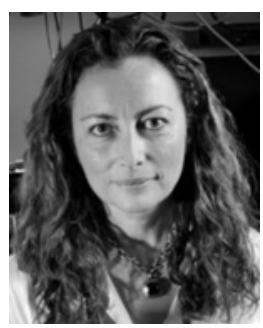

Adela Ben-Yakar received the B.Sc. (cum laude) and the M.S. degrees in 1995 from The TechnionIsrael Institute of Technology, Haifa, Israel, and the $\mathrm{Ph} . \mathrm{D}$. degree in engineering from Stanford University, Stanford, CA, USA, in 2000. She completed her postdoctoral work at Stanford University, in applied physics, and Harvard University in physics in 2004 She is currently an Associate Professor in the Mechanical Engineering Department and a member of the Graduate Student Council in the Electrical and Computer Engineering and Biomedical Engineering Departments. Her research interests include ultrafast laser microsurgery, plasmonic laser nanosurgery, nonlinear imaging, clinical image-guided surgery endoscopes, and high-throughput opto-fluidics systems for drug screening using small animal models as applied to nerve regeneration, Alzheimer's disease, and aging. Her work has been published in high impact journals and extensively covered in press. She is the recipient of the Fulbright Scholarship, Zonta Amelia Earhart Award, NSF Career Award, Human Frontier Science Program Research Award, and NIH Director's Transformative Award. She is a member of OSA and SPIE. 